\title{
1 Single-cell atlas of the first intra-mammalian developmental stage 2 of the human parasite Schistosoma mansoni
}

4 Carmen Lidia Diaz Soria ${ }^{1} \#$, Jayhun Lee ${ }^{2}$, Tracy Chong ${ }^{2,3}$, Avril Coghlan ${ }^{1}$, Alan Tracey ${ }^{1}$, 5 Matthew D Young ${ }^{1}$, Tallulah Andrews ${ }^{1}$, Christopher Hall ${ }^{1}$, Bee Ling $\mathrm{Ng}^{1}$, Kate Rawlinson ${ }^{1}$, 6 Stephen R. Doyle ${ }^{1}$, Steven Leonard ${ }^{1}$, Zhigang Lu${ }^{1}$, Hayley M Bennett ${ }^{1}$, Gabriel Rinaldi ${ }^{1 *}$, $7 \quad$ Phillip A. Newmark ${ }^{2,3 *}$, Matthew Berriman ${ }^{1 *}$.

\section{Affiliations}

91 Wellcome Sanger Institute, Wellcome Genome Campus, Hinxton, Cambridgeshire, UK

102 Regenerative Biology, Morgridge Institute for Research, Madison, WI, USA

113 Howard Hughes Medical Institute, Department of Integrative Biology, University of 12 Wisconsin-Madison, Madison, WI, USA

13 \#Equal contribution: Carmen L. Diaz Soria, Jayhun Lee

14 *Co-corresponding authors: Gabriel Rinaldi (gr10@sanger.ac.uk), Phillip A. Newmark 15 (PNewmark@morgridge.org), and Matthew Berriman (mb4@sanger.ac.uk)

17 Abstract

Over 250 million people suffer from schistosomiasis, a tropical disease caused by parasitic flatworms known as schistosomes. Humans become infected by free-swimming, water-borne larvae, which penetrate the skin. The earliest intra-mammalian stage, called the schistosomulum, undergoes a series of developmental transitions. These changes are critical for the parasite to adapt to its new environment as it navigates through host tissues to reach its niche, where it will grow to reproductive maturity. Unravelling the mechanisms that drive intra-mammalian development requires knowledge of the spatial organisation and transcriptional dynamics of different cell types that comprise the schistomulum body. To fill these important knowledge gaps, we performed single-cell RNA sequencing on two-day old schistosomula of Schistosoma mansoni. We identified likely gene expression profiles for muscle, nervous system, tegument, parenchymal/primordial gut cells, and stem cells. In 
addition, we validated cell markers for all these clusters by in situ hybridisation in schistosomula and adult parasites. Taken together, this study provides a comprehensive celltype atlas for the early intra-mammalian stage of this devastating metazoan parasite.

\section{Introduction}

Schistosomes are parasitic flatworms that cause schistosomiasis, a serious, disabling, and neglected tropical disease (NTD). More than 250 million people require treatment each year, particularly in Africa ${ }^{1}$. The life cycle of this metazoan parasite is complex. A schistosome egg hatches in water to release a free-living, invasive larva that develops into asexually replicating forms within aquatic snails (the intermediate host). From the snail, thousands of cercariae -a second free-living larval form- are released into freshwater to find and invade a mammal (the definitive host). In the mammalian host, the larvae (schistosomula) migrate and develop into distinctive male or female adult worms ${ }^{2}$ (Figure 1A). While the only drug currently available to treat schistosomiasis (praziquantel) works efficiently to kill adult parasites, it is less effective against immature parasites including schistosomula ${ }^{3}$. Understanding the parasite biology is a critically important step for developing novel strategies to treat and control this NTD.

During invasion, the parasite undergoes a major physiological and morphological transformation from the free-living, highly motile cercariae to the adult parasitic form ${ }^{2}$. Upon penetration, the tail used for swimming is lost. Less than three hours after entering the host, the thick glycocalyx is removed and the tegument is remodelled to serve both nutrient-absorption and immune-protection roles ${ }^{4}$. Throughout the rest of the organism's life in the definitive host, a population of sub-tegumental progenitor cells continuously replenish the tegument, allowing the parasite to survive for decades ${ }^{5,6}$. The schistosomula make their way into blood or lymphatic vessels and, one week after infection, reach the lung capillaries ${ }^{7}$. The migration through the lung requires coordinated neuromuscular activities, including cycles of muscle elongation and contraction ${ }^{8}$, to squeeze through capillaries and reach the general circulation ${ }^{7}$. Over the following weeks, the parasites mature further into sexually reproducing adults. Dramatic changes to the parasite are required that include posterior growth, remodelling of the musculature $^{9}$ and nervous system ${ }^{10,11}$ as well as the development of the gonads ${ }^{12}$ and gut $^{13}$. This extensive tissue development starts in the schistosomula, with stem cells driving these transitions ${ }^{6,14,15}$. However, to decipher cellular and molecular mechanisms underlying 
60 schistosomula development, a detailed understanding of the spatial organisation and

61 transcriptional programs of individual cells are needed.

62 Important insights into major processes that underlie the transformations across the life cycle 63 have been gained from bulk transcriptomic studies ${ }^{5,6,14-24}$. However, these studies are not able 64 to quantify the relative abundance of different cell types from the absolute expression per cell, 65 and the signal from highly expressed genes in a minority of cells can often be masked by a 66 population averaging effect. Single-cell RNA sequencing has previously been used 67 successfully to characterise cell types ${ }^{25-32}$ and understand how the cell expression profile 68 changes during differentiation ${ }^{31-38}$. Notable examples include recent studies in the free-living 69 planarian flatworm Schmidtea mediterranea ${ }^{31,32,39}$, a well-established model to study regeneration in the Phylum Platyhelminthes ${ }^{40}$.

71 Here, we have used scRNAseq to characterise two-day schistosomula obtained by in vitro transformation of cercariae ${ }^{22}$ using $10 \mathrm{X}$ Chromium technology and validated the cell clusters by RNA in situ hybridization (ISH) in schistosomula and adult worms. We identified eleven discrete cell populations, and described and validated novel marker genes for muscles, nervous system, tegument, parenchymal/gut primordia and stem cells. This study lays the foundation towards a greater understanding of cell types and tissue differentiation in the first intramammalian developmental stage of this NTD pathogen. 


\section{Results}

\section{Identification of 11 transcriptionally distinct cell types in schistosomula.}

We performed single-cell RNA sequencing of schistosomula collected two days after mechanically detaching the tail from free-living motile larvae (cercariae) (Figure 1A). To do so, we first developed a protocol to efficiently dissociate the parasites using a protease cocktail, after which individual live cells were collected using fluorescence-activated cell sorting (FACS) (Figure 1A and Supplementary Figure 1A). Using the droplet-based 10X Genomics Chromium platform, we generated transcriptome-sequencing data from a total of 3,513 larval cells, of which 2,144 passed strict quality-control filters, resulting in a median of 900 genes and depth of 283,000 reads per cell (Table S1). Given that an individual schistosomulum comprises $\sim 900$ cells (Supplementary Figure 1B), the number of quality-controlled cells theoretically represents $>2 x$ coverage of all cells in the organism at this developmental stage.

To create a cellular map of the $S$. mansoni schistosomula, we used a combination of the $\mathrm{SC}^{41}$, Seurat ${ }^{42}$ and $\mathrm{UMAP}^{43}$ algorithms to cluster cells based on their mRNA expression levels and statistically identify marker genes that were best able to discriminate between the clusters (Figures 1B and 1C, Table S2-S5). To identify which cells each cluster represented, we curated gene set lists of previously defined cell-specific markers (Table S6). For example, tegument ${ }^{5,6,44-46}$ and stem ${ }^{14,47-49}$ cell clusters were identified based on known marker genes in S. mansoni, whereas muscle cells ${ }^{50-52}$ and neurons ${ }^{53-55}$ were identified based on characterised marker genes in mouse and humans (Table S6). Based on the marker genes identified using Seurat, we identified three distinct muscle-like clusters composed of 1,105 cells, two apparent tegumental clusters (253 cells), two parenchymal clusters (155 cells), one cluster resembling stem cells (94), three resembling the nervous system (311 cells), and one ambiguous cluster of 226 cells that could not be experimentally defined. Gene Ontology (GO) analysis of the marker genes generally matched the predicted cellular processes for each cluster (Supplementary

104 Figure 1C). For instance, as expected, the stem/germinal cell cluster showed a significant enrichment in genes involved in translation, DNA replication, and RNA binding. Meanwhile, neuronal cells and muscle cells were enriched in processes involved in GPCR signalling and cytoskeleton, respectively. These analyses suggested that each cluster is molecularly distinct and likely display different biological functions. Therefore, we defined highly specific cluster- 
defining transcripts (potential cell markers) and characterised their spatial expression in both larval schistosomula and adult schistosomes by ISH (Table S7).

\section{Muscle cells show position dependent patterns of expression}

112 Three discrete muscle clusters were identified by examining the expression of the well113 described muscle-specific genes $\operatorname{myosin}^{56}$ and $\operatorname{troponin}^{52}$ (Figure 2A). One muscle cluster

114 showed high expression of the uncharacterised gene Smp_161510, which was expressed along 115 the dorso-ventral axis of two-day old schistosomula (Figure 2B). In adult worms, Smp_161510 116 exhibited no dorsal-ventral expression pattern; instead, Smp_161510 expression was scattered 117 throughout the worm body (Supplementary Figures 2A and 2B). A subset of cells in this muscle 118 cluster expressed wnt (Smp_167140) (Figure 2A). These wnt + cells showed an anterior119 posterior gradient in two-day schistosomula (Figure 2C) that remained consistent during the 120 development from juveniles to mature adult worms (Figure 2D and Supplementary Figure 2A). 121 Given that these markers have been shown to have distinct spatial distributions ${ }^{57,58}$, we decided 122 to term this population 'positional muscle'.

123 A second muscle-like cluster was uniquely found to express genes encoding a rhodopsin 124 orphan GPCR (Smp_153210) (Figure 2E) and an ortholog of the myoD transcription factor 125 (Smp_167400) from S. mediterranea (dd_Smed_v6_12634_0_1) $)^{31}$. Both genes showed a 126 scattered expression pattern throughout the schistosomula (Figure 2E), and adult body (Figure 2F, Supplementary Figure 2B).

128 Finally, the third cluster of putative muscle cells was shown to be enriched in actin-2 129 (Smp_307020,SSmp_307010) expression. FISH confirmed actin-2 expression throughout the 130 body of the schistosomula (Figure 2A). Our transcriptomic data suggested that actin-2 was 131 enriched but not specific to this cluster. In line with the transcriptome evidence, ISH revealed 132 that actin-2 is also expressed in some cells of both the 'positional muscle' and myoD+ 133 populations in schistosomula (Figures 2C, 2E-F) and in adults (Supplementary Figure 2C).

134 Together, we identified three transcriptionally distinct cell types validated by ISH that represent schistosomula muscle cells (Figure $2 \mathrm{H}$ ).

\section{Schistosomula have two distinct populations of tegumental cells}

137 We identified two populations of tegumental cells (Tegument 1 and Tegument 2, Figure 3A).

138 The first tegumental cluster (Tegument 1) expressed several known tegument genes, including 
139 four that distinguish it from Tegument 2 and encode: Fimbrin (Smp_037230), TAL10

140 (Smp_074460), Annexin B2 (Smp_077720) and Sm21.7 (Smp_086480) (Figure 3A; Table

141 S6 $)^{6,45,46,59}$.

142 The tegument 1 population also showed enrichment for an uncharacterised gene (Smp_022450)

143 that, to our knowledge, has not previously been reported as a tegument-associated gene. We

144 found that cells in the head, neck and body of the schistosomulum that expressed Smp_022450

145 co-localised with the tegument marker annexin B2 (Smp_077720) (Figure 3B). In addition,

146 cells expressing annexin B2 and Smp_022450 were dextran+ (Figure 3C, Supplementary

147 Figure 3A). Fluorescently conjugated dextran specifically labels tegumental cell bodies ${ }^{6}$, thus

148 further validating Smp_022450 as a tegumental marker. In addition, Tegument 1 showed

149 enrichment for microexon genes meg3 (Smp_138070) and meg17 (Smp_180620). The

150 microexon gene meg3 co-localised with the novel tegument gene Smp_022450 in the neck and

151 anterior region of the larva (Figure 3D). The gene meg17 was expressed in the neck and

152 oesophageal region (Figure 3E). Given the expression of some meg genes in the oesophagus of 153 adult male and female parasites ${ }^{60}$ and the developmental relationship between the oesophagus 154 and the tegument ${ }^{8,61}$, we tested if meg17 co-localised with any known oesophageal marker. We 155 found that cells expressing meg 17 also expressed the known oesophageal marker meg $4^{62}$ 156 (Smp_163630) (Figure 3E). These results suggest that a subset of Tegument 1 cells likely 157 represent primordial oesophageal gland cells.

158 Distinguishing the second tegumental cluster was challenging due a lack of Tegument 2159 specific markers (Figure 3A). Two genes - ccdc74 (Smp_030010) and nmda (Smp_181470) 160 with similarly enriched expression in both clusters were selected for further investigation. 161 Double FISH experiments using either $c c d c 74$ (Smp_030010) or nmda (Smp_181470) with 162 Smp_022450 showed co-localisation of expression (Figure 3F-G). In addition, these cells were 163 also dextran $+^{6}$, confirming their tegumental assignment (Supplementary Figure 3B and 3C). In 164 adults, marker genes of Tegument 1 and 2 showed overall similar enriched expression patterns 165 in the anterior cell mass, ventral sucker, and tegumental cells throughout the worm body 166 (Supplementary Figures 3D and 3E).

167 To explore more subtle differences in expression profiles between these two tegumental 168 populations, we investigated tentative functional differences. Analysis of marker genes for 169 Tegument 2 using the STRING database predicted a group of interacting genes involved in 170 clathrin-mediated endocytosis ${ }^{63}$ (Supplementary Figure 3F-3H). This group included genes 
that encode Phosphatidylinositol-binding clathrin assembly protein (Smp_152550), Epsin15related (Smp_171640) and Epsin4 (Smp_140330) proteins. The potential involvement of

173 Tegument 2 cells in calcium binding (Supplementary Figure 1C) and clathrin-mediated endocytosis is consistent with previous studies showing that numerous vesicles are produced by endocytosis from cell bodies and trafficked to the syncytial cytoplasm of the tegument ${ }^{64,65}$. Together, the evidence provided here supports these cells being part of the schistosomulum tegument (Figure 3H).

\section{Identification of schistosome parenchymal and primordial gut cells}

179 Schistosomes, like other platyhelminths, are acoelomates and lack a fluid-filled body cavity.

180 Instead, their tissues are bound together by cells and extra-cellular matrix of the parenchyma ${ }^{20}$.

181 We identified two cell types that most likely represent parenchymal cells (Parenchymal 1 and

182 2) that showed enriched expression of numerous enzymes such as lysosome, peptidase, and 183 cathepsin (Figure 4A).

184 Cells expressing cathepsin B (Smp_141610) were spread throughout the worm parenchyma 185 and showed long cytoplasmic processes stretching from each cell (Figure 4B-C and 186 Supplementary Figure 4D-E). A similar expression profile was observed for serpin 187 (Smp_090080) expressing cells in the later stages of schistosomula as well as in adult parasites 188 (Supplementary Figures 4A-4C). In addition, parenchymal cells did not co-express other cell 189 type markers except for actin-2, which showed slightly overlap in expression (Supplementary $1904 \mathrm{~F}, 4 \mathrm{~J})$.

191 In Parenchymal 2 cells, we found that leucine aminopeptidase (lap) (Smp_030000) was 192 expressed in the primordial gut (cathepsin $B^{\prime}(+)$ and surrounding parenchymal tissue (Figure 193 4D). Such mixed gut/parenchymal expression was also observed in adult parasites (Figure 4E, 194 Supplementary Figure 4B). This is consistent with previous studies in adult parasites where 195 LAP was detected in the gut and in cells surrounding the gut ${ }^{66}$. Overall, the identified genes 196 that mark schistosomula parenchyma, while a few of them are also expressed in the gut 197 primordia (Figure 4F).

\section{Stem cells in two day old schistosomula}

199 Recently, it was shown that schistosomula carry two types of stem cell populations: somatic stem cells and germinal cells ${ }^{15}$. The somatic stem cells are involved in somatic tissue 
201

202

203

204

205

206

207

208

209

210

211

212

213

214

215

216

217

218

219

220

221

222

223

224

225

226

227

differentiation and homeostasis during the parasite intra-mammalian development, whereas the germinal cells are presumed to give rise to germ cells (sperm and oocytes) in adult parasites ${ }^{15}$. Less than 24 hours after the cercaria enters the mammalian host to become schistosomulum, $\sim 5$ somatic stem cells at distinct locations begin to proliferate ${ }^{15}$ (Figure 5B). Germinal cells, on the other hand, are thought to be packaged in a distinct anatomical location called the germinal cell cluster, and only begin to proliferate $\sim 1$ week after penetrating the host ${ }^{15}$.

We identified a single stem/germinal cell cluster that expressed the canonical cell cycle markers histone h2a (Smp_086860) ${ }^{15}$ and histone h2b (Smp_108390) ${ }^{6}$ (Figure 5A). In addition, this cluster also had a significant enrichment of translational components (Supplementary Figure 1C). We confirmed that histone h2a (Smp_086860) is expressed in $~ 5$ cells, 1 medial and 2 sets on each side (Figure 5B) and also in the germinal cell cluster a few days later (Supplementary Figure 5A). In adults, histone h2a (Smp_086860) is expressed in somatic cells as well as in cells of the gonads (testis, ovary, and vitellaria) (Supplementary Figure 5B). In addition, we identified a novel stem/germ cell marker calmodulin (cam) (Smp_032950). This gene was expressed similarly to $h 2 a$, but in some schistosomula, a few more cam+ cells could be observed medially as well as near the germinal cell cluster (Figure $5 \mathrm{C}$ ). The cam + cells were also positive for $h 2 b$ (Figure 5D), and found to be expressed in the adult gonads (Figure 5E) and in adult soma (Figure 5F).

In addition to histone $22 a$ (Smp_086860), histone h2b (Smp_108390) and cam (Smp_032950), cells in this cluster expressed stem cell markers including fgfrA (Smp_175590) and nanos-2 (Smp_051920) ${ }^{14,15,67}$ (Figure 5A). Given that many of these genes have been associated with two distinct stem cell populations ${ }^{15}$ (somatic and germinal), we tested if these cells could be further subclustered, but were unable to do so, presumably due to the low expression level of some of these genes in most cells in this cluster (Supplementary Figure 5C). Overall, these data suggest that this cluster does indeed represent population(s) of stem cells that might give rise to somatic and germ cells during the course of parasite development within the mammalian host (Figure 5G). 
Platyhelminths have a central nervous system comprised of cephalic ganglia and main nerve cords, and a peripheral nervous system with minor nerve cords and plexuses ${ }^{10}$. This system also plays a neuroendocrine role by releasing neuromodulators during development and growth $^{10,68,69,70}$.

233 We identified three distinct populations that expressed neural-associated genes (Figure 6A).

234 One population was characterised by the expression of genes encoding neuroendocrine protein 7B2 (7b2, Smp_073270) and neuroendocrine convertase 2 (pc2, Smp_077980) and lack of gnai (Smp_246100) expression (Figure 6A). The in situ hybridisation of $7 b 2$ (Smp_073270) showed expression in cells of the cephalic ganglia in schistosomula (Figure 6B-C). The cephalic ganglia region was identified using lectin succinylated Wheat Germ Agglutinin (sWGA) ${ }^{11}$ staining. In adult worms, $7 b 2$ was expressed in the cephalic ganglia as well as in the main and minor nerve cords (Figure 6C). We refer to this cluster as ' $7 b 2 / p c 2+$ nerve' cells.

241 A second population expressed the uncharacterised gene Smp_203580 (Figure 6D). Co-

242 localisation experiments with $7 b 2$ confirmed that this population was distinct from the central ganglia population (Figure 6D). In the larvae, only six cells (two cells in the head and four cells in the body) expressed the novel marker Smp_203580 (Figure 6D) but in adults, an expanded number of cells were found throughout the body of the parasite (Supplementary Figures 6A and 6C). These cells displayed 2-3 long cellular processes, branching into different directions

247 (Supplementary Figure 6B). Interestingly, cells in this cluster also expressed the marker gene encoding KK7 (Smp_194830), known to be associated with the peripheral nervous system in S. mansoni $^{55}$ (Figure 6E, and Supplementary Figures 6A and 6D). Therefore, we refer to this population as ' $S m-k k 7+$ nerve cells'.

251 Finally, we identified a population of cells that expressed gnai (Smp_246100), a gene encoding 252 a G-protein G(i) alpha protein. FISH experiments showed expression of this gene in three cells: 253 one in gland region of the head, one in the neck region, and one in the body region (Figure 6F).

254 In adults, this gene is expressed around the main and minor nerve cords (Figure 6G and 255 Supplementary Figure 6E and 6F). Some gnai + cells are also 7b2+ (Figure 6G). We designated 256 this population as 'gnai+ neurons'. Overall, neuronal cells are transcriptionally and spatially heterogeneous (Figure $6 \mathrm{H}$ ) and thus are expected to be involved in diverse biological processes. 
258 Conserved gene expression patterns in stem cells and neurons between $\boldsymbol{S}$. mansoni and

259 Schmidtea mediterranea

260 Given that some of the populations described herein had not been previously characterised, we

261 asked if we could further annotate our dataset by comparison to previously annotated single-

262 cell RNAseq data from Schmidtea mediterranea, the closest free-living model organism to $S$.

263 mansoni $^{31}$. To compare clusters, we used a random forest (RF) model trained on $S$.

264 mediterranea to map gene expression signatures between both datasets ${ }^{71}$. Using the RF model,

265 we classified each of the larval S. mansoni cells using the adult Schmidtea labels. We

266 discovered that the stem cell population in our dataset mapped to Schmidtea stem cells (Figure

267 7). This is consistent with previous work that showed similarities between Schmidtea and $S$.

268 mansoni stem cells ${ }^{5,14,15,49,67,72}$. We found that $S m-k k 7+$ cells in schistosomula mapped to the

269 neuronal population annotated as otoferlin $1($ otfl $1+)$ cells described by Plass et. al ${ }^{31}$. In

270 addition, $7 b 2 / p c 2+$ cells in $S$. mansoni mapped to spp $11+$ and Chat neurons as well as neural

271 progenitors in Schmidtea (Figure 7). In addition, tegument clusters in S. mansoni mapped to

272 early and late epidermal progenitors in Schmidtea. The rest of the clusters in S. mansoni were

273 labelled as $p s d+$ cells (of unknown function in $S$. mediterranea) and neoblasts. Taken together,

274 these results suggest that despite great differences in developmental stages between larval

275 schistosomula and the asexual adult Schmidtea mediterranea used for this comparison, marker

276 genes for stem cells and neuronal populations have been conserved (Table S8-S10). 


\section{Discussion}

278 In this study, we have generated a cell atlas of the schistosomulum, the first intra-mammalian

279 developmental stage of S. mansoni and a key target for drug and vaccine development ${ }^{73,74}$. Our

280 transcriptome analysis enabled the characterisation of 11 distinct clusters, with sufficient

281 sensitivity to detect as few as three cells per parasite, as demonstrated by the ISH experiments

282 (Figure 6F). Importantly, the latter allowed us to validate key marker genes for each of the cell

283 clusters, spatially mapping the cell populations in both schistosomula and adult worms and

284 linking transcriptomic profiles to anatomical features of the organism.

285 By determining the transcriptome of individual cells from schistosomula, we uncovered marker genes not only for known populations, such as stem and tegument cells, but also for previously undescribed cell clusters, such as parenchymal cells. We found that marker genes of the parenchymal tissue are also expressed in the primordial gut. However, the relationship between the parenchyma and gut primordial cells is yet to be determined. In planarians, the orthologous cathepsin gene (dd_Smed_v6_81_0_1) is a marker for cathepsin+ cells that include cells in the parenchyma $^{32}$. This planarian cathepsin (dd_Smed_v6_81_0_1) is also expressed in the intestine $^{32}$ and gut phagocytes ${ }^{31}$. Similarly, planarian aminopeptidase (dd_Smed_v6_181_0_1) is expressed in cathepsin+ cells, epithelia and intestine ${ }^{31,32}$. Thus, further work is required to characterise schistosome parenchymal cells and their signaling mechanisms with the surrounding gut cells ${ }^{75}$.

296 Until now, S. mansoni cell types have been revealed primarily through a combination of morphological and ISH studies of specific tissues, with stem and tegument cell populations being among the best characterised ${ }^{5,6,14,15,49}$. In the present study, we identified and validated a novel stem cell marker calmodulin (Smp_032950) that, to our knowledge, has not previously

300 been associated with stem cells. Calmodulins are $\mathrm{Ca}^{2+}$ transporters required for the miracidiumto-sporocyst transition, sporocyst growth ${ }^{76}$ and egg hatching ${ }^{77}$. In addition, we found this gene to be expressed in the reproductive organs of adult males and females.

303 Coordinated neuromuscular activity is essential for schistosomes to migrate through host 304 tissue $^{78}$. Although circular and longitudinal muscle layers have been described in S. mansoni ${ }^{9,11,78}$, we found no evidence that the three muscle clusters correspond to different anatomical fiber arrangements. In the free-living planarian $S$. mediterranea, a population of muscle cells also shows no specific muscle layer localisation, but instead forms a cluster based 
on enriched expression of position-control genes (PCGs) ${ }^{32,79}$. We therefore reasoned that this may be the case for at least some of the muscle cells in our dataset.

310 Knowledge of planarian stem cells has previously informed the study of stem cells in

$311 S$. mansoni ${ }^{67}$. Our comparison between schistosomula and S. mediterranea clusters uncovered

312 conserved features for stem cells and neurons and served to support cell type assignment in

313 schistosomula. Given that nerve cell populations have remained poorly characterised at the

314 transcriptome level in schistosomes, planarians may serve as a model to understand the nervous

315 system biology in schistosomula. A particularly attractive feature of the planaria biology is the

316 remarkable regenerative properties of the these worms. An individual worm comprises all cell

317 types at intermediate stages of development and regeneration $31,32,80$. This has enabled recent

318 single-cell sequencing studies in planarians to characterise developmental trajectories from

319 within the soma of adult worms ${ }^{31}$. However, schistosomes do not share this regenerative

320 property with their distant free-living relatives, instead intermediate stages of schistosome

321 development necessarily need to be captured. The data from the present study represent the

322 first logical step in that characterisation.

323 Despite having successfully characterised several previously unknown marker genes and 324 populations, we faced challenges throughout the course of this study. Some cells were not detected, possibly because they are difficult to isolate or relatively rare. One notable example was the absence of eight known protonephridia cells in the parasite at this developmental stage $^{11,81}$. Previous single-cell studies in $S$. mediterranea have found that relatively rare cell types are sometimes embedded in larger neuronal clusters ${ }^{31,32}$, and therefore, it is possible that this is also the case for this cell group. In addition, schistosomula obtained for this study were a mixture of males and females. While the male and female schistosomula are morphologically identical, they may bear transcriptomic differences that are important for early stages of reproductive development ${ }^{82}$. Future scRNA-Seq studies obtained separately from male and female schistosomula will be needed to resolve this question.

334 Our study demonstrates the power of single-cell sequencing, coupled with ISH validation, to 335 transcriptionally and spatially characterise cell types of an entire metazoan parasite for the first 336 time. This approach is essential for unravelling the developmental biology of this important 337 parasite. 


\section{Ethics statement}

340 The complete life cycle of Schistosoma mansoni (NMRI strain) is maintained at the Wellcome

341 Sanger Institute (WSI). The mouse infections at WSI were conducted under Home Office

342 Project Licence No. P77E8A062 held by GR, and all protocols were presented and approved

343 by the Animal Welfare and Ethical Review Body (AWERB) of the WSI, and Institutional

344 Animal Care and Use Committees (IACUC) at the University of Wisconsin-Madison (protocol

345 M005569). The AWERB is constituted as required by the UK Animals (Scientific Procedures)

346 Act 1986 Amendment Regulations 2012.

\section{Preparation of parasites}

348 S. mansoni schistosomula were obtained by mechanical transformation of cercariae and 349 cultured as described previously ${ }^{83}$. In brief, snails were washed, transferred to a beaker with 350 water $(\sim 50-100 \mathrm{ml})$ and exposed under light to induce cercarial shedding for two hours, 351 replacing the water and collecting cercariae every $30 \mathrm{~min}$. Cercarial water collected from the 352 beaker was filtered through a $47 \mu \mathrm{m}$ stainless steel Millipore screen apparatus into sterile 50 353 ml-Falcon tubes to remove any debris and snail faeces. The cercariae were concentrated by 354 centrifugation ( $800 \mathrm{~g}$ for $15 \mathrm{~min}$ ), washed three times in 1X PBS supplemented with 2\% PSF 355 (200 U/ml penicillin, $200 \mu \mathrm{g} / \mathrm{ml}$ streptomycin, $500 \mathrm{ng} / \mathrm{ml}$ amphotericin B), and three times in 356 'schistosomula wash medium' (DMEM supplemented with 2\% PSF and 10 mM HEPES (4-(2357 hydroxyethyl)-1-piperazineethanesulfonic acid)). The cercarial tails were sheared off by $\sim 20$ 358 passes back and forth through a 22-G emulsifying needle, schistosomula bodies were separated 359 from the sheared tails by Percoll gradient centrifugation, washed three times in schistosomula 360 wash medium and cultured at $37^{\circ} \mathrm{C}$ in modified Basch's medium under $5 \% \mathrm{CO}_{2}$ in air ${ }^{83}$.

\section{$361 \quad$ Single-cell tissue dissociation}

362 Two days after transformation the schistosomula cultured in modified Basch's media at $37^{\circ} \mathrm{C}$ 363 and 5\% $\mathrm{CO}_{2}$ were collected and processed in two separate batches (batch1 and batch2). 364 Schistosomula collected from two different snail batches were considered biological replicates.

365 Data collected as batch3 are 'technical' replicates of batch2 given they were collected on the same day and from the same pool of parasites. In each experiment, approximately 5,000 larvae 
at $300 \mathrm{rpm}$ at $37^{\circ} \mathrm{C}$, using a digestion solution of $750 \mu \mathrm{g} / \mathrm{ml}$ Liberase DL (Roche 05466202001 ). The resulting suspension was passed through $70 \mu \mathrm{m}$ and $40 \mu \mathrm{m}$ cells strainers (Falcon) .

370 Dissociated cells were spun at $300 \mathrm{rpm}$ for $5 \mathrm{mins}$ and resuspended in $1 \mathrm{X}$ cold PBS supplemented with $20 \%$ heat inactivated fetal bovine serum (twice). The resulting cell suspension was co-stained with $0.5 \mu \mathrm{g} / \mathrm{ml}$ of Fluorescein Diacetate (FDA; Sigma F7378) to label live cells, and $1 \mu \mathrm{g} / \mathrm{ml}$ of Propidium Iodide (PI; Sigma P4864) to label dead/dying cells, and sorted into eppendorf tubes using the BD Influx ${ }^{\mathrm{TM}}$ cell sorter by enriching for FDA+/ PIcells ${ }^{84}$. It took 2-3 hours from the enzymatic digestion to generating single-cell suspensions ready for library preparation on the 10X Genomics Chromium platform.

\section{X Genomics library preparation and sequencing}

378 The concentration of single cell suspensions was approximately 500 cells $/ \mu 1$ as estimated by

379 flow cytometry-based counting. Cells were loaded according to standard protocol of the

380 Chromium single-cell 3' kit in order to capture approximately 7,000 cells per reaction (V2 chemistry). However, after sequencing and preliminary analysis, we found the actual number of captured cells was closer to $\sim 1200$ cells per experiment. Single-cell libraries were sequenced on an Illumina Hiseq4000 (paired-end reads 75bp), using one sequencing lane per sample. All raw sequence data is deposited in the ENA under the project accession ERP116919.

\section{Protein-coding genes}

$386 S$. mansoni gene annotation is based on the latest genome assembly (v7, unpublished). The identifier for all genes contains the Smp_prefix followed by a unique 6-digit number; entirely new gene models have the first digit ' 3 ', eg. Smp_3xxxxx. To assign a gene name and functional annotation (used in Tables S4-S6) to 'Smp_' identifiers, protein-coding transcript sequences were blasted against SwissProt3 to predict product information (blastp v2.7.0). Some genes also maintained previous functional annotation from GeneDB. Genes lacking predicted product information were named hypothetical genes.

\section{Mapping and quantification of single-cell RNA-seq}

394 Single-cell RNA-seq data were mapped to the S. mansoni reference genome version 7 395 (https://parasite.wormbase.org/Schistosoma_mansoni_prjea36577) using the 10X Genomics analysis pipeline Cell Ranger (v 2.1.0). The default cut-off provided by Cell ranger was used 
transcriptome with an average 297,403 reads per cell. In total 3,513 cells were sequenced, with a median 918 genes expressed per cell.

\section{Quality control of single-cell data}

401 To filter lower quality cells, the best practices for pre-processing and quality control from the

402 Scater package (version 1.8.4) ${ }^{85}$ were followed. We first created a single cell experiment using 403 SingleCellExperiment: S4 Classes for Single Cell Data R package version 1.5.0 ${ }^{86}$. Cells that 404 had greater than 30,000 Unique Molecular Identifiers (UMIs) were removed. Although tools 405 are not currently available to determine biological doublets, at the concentrations of cells used 406 in these experiments, the doublet rate is expected to be very low $(\sim 1 \%)$. In addition, cells with 407 mitochondrial gene expression greater than $3 \%$ or cells that expressed fewer than 600 genes 408 per cell were excluded.

409 We further filtered the data by generating a consensus matrix with the SC3 package (version $4101.8 .0)^{41}$ and excluded any clusters with a cluster stability index of less than 0.10 . This was done 411 on the basis that cells with low stability index included cells that could not be assigned 412 confidently to a specific cell population. We also excluded clusters containing less than 3 cells 413 due to the limitations of SC3 to capture rare cell types ${ }^{41}$. In total 2,144 cells out of 3,513 cells 414 passed QC. Further exclusion of one ambiguous cluster resulted in a total of 1,918 cells.

\section{Data normalisation}

416 Data was first clustered with the quickCluster function from scran (version 1.8.4) ${ }^{87}$. The 417 quickCluster function groups cells according to their expression profiles. Cell biases were 418 normalised using the computeSumFactors function. The computeSumFactors function works 419 on the assumption that most genes are not differentially expressed between cells. As such, any 420 differences in expression across the majority of the genes are the result of technical biases in 421 the single-cell dataset and need to be removed ${ }^{87}$. Finally, the normalised expression values 422 were calculated using the normalise function from the scater package (version 1.8.4) ${ }^{85}$.

\section{Clustering and QC using SC3}

424 The SC3 package (version 1.8.0) was used to cluster and exclude low quality cells from the 425 dataset $^{41}$. For the consensus clustering, SC3 uses the consensus-based similarity partitioning 426 algorithm (CSPA). SC3 constructs a binary similarity matrix using cell labels. When two cells 
belong to the same cluster, the assigned value is 1 ; otherwise the value is 0 . A consensus matrix is the result of the averaging of all similarity matrices of individual clustering. Based on the consensus matrix, the cells were then clustered using hierarchical clustering using $k$ levels of

430 hierarchy where $k$ was specified. In the first instance we used a range of values close to the $k$

431 value estimated using the sc3_estimate_k function $(k=26)$ from the SC3 package. The stability 432 and quality of the clusters was assessed by visually inspecting the data obtained for the 433 specified $k$ value ranges. Clusters with stability index less than 0.10 and/ or less than 3 cells were excluded from further analysis. We continued to re-cluster cells until all clusters had stability values greater than 0.6 and contained more than 5 cells. We also sub-clustered populations of cells that were contained within the same $k$ level of hierarchy but appeared to be distinct subpopulations of cells.

\section{Clustering using Seurat after QC steps}

439 The Seurat package (version 2.3.4) (https://satijalab.org/seurat/) was used to analyse the raw 440 values of QC matrix ${ }^{42}$. First, we normalised using the NormalizeData function from Seurat 441 (http://satijalab.org/seurat/). Following normalisation, we identified highly variable genes 442 using the Seurat FindVariableGenes function using the cut-offs stated in the website: $z=0.5$ and 443 mean expression in the range 0.0125 to 3 . We identified 12 clusters (including the ambiguous 444 cluster) using the FindClusters function from Seurat with a resolution of 0.6.

\section{Identifying marker genes and cluster annotation}

446 To annotate each cluster, we manually inspected the top markers for each of the populations 447 and compared to the top markers curated from the literature (Table S6). We used the top 448 markers identified by SC3 and Seurat packages. SC3 identifies marker genes for each cluster 449 by constructing a binary classifier based on the mean expression values for each gene. The area 450 under the operating characteristic (ROC) curve is used to quantify the confidence for that 451 specific marker. A Wilcoxon signed-rank test is used to assign a P-value to each gene. We 452 relied on high quality marker genes with area under the curve (AUROC) $>0.8, P<10^{-5}$ and spatial information of those genes to determine the identity of a specific population. We also used the Seurat package to identify marker genes for each population using the function FindAllMarkers, using the likelihood ratio as specified in the Seurat best practices 


\section{Gene Ontology (GO) analysis}

458 The Gene Ontology (GO) annotation for S. mansoni was obtained using InterProScan v5.25-

45964.0 (https://www.ebi.ac.uk/interpro/). GO term enrichment was performed using the weight01

460 method provided in topGO ${ }^{88}$ v2.34.0 (available at

461 http://bioconductor.org/packages/release/bioc/html/topGO.html) for all three categories (BP,

$462 \mathrm{MF}$, and CC). For each category, the analysis was restricted to terms with a node size of $>=5$.

463 Fisher's exact test was applied to assess the significance of overrepresented terms compared 464 with all expressed genes. The threshold was set as FDR $<0.01$.

\section{STRINGdb Analysis}

466 We used STRINGdb ${ }^{89}$ to identify possible gene interactions that would enable us to 467 differentiate between tegumental clusters. Briefly, the $S$. mansoni V7 gene identifiers for the 468 tegument 2 cluster with AUROC $\geq 0.7$ in Seurat were converted to $S$. mansoni V5 gene 469 identifiers. The V5 gene identifiers were analysed in STRINGdb v11.089. Human, 470 Caenorhabditis elegans and Drosophila melanogaster orthologs of these genes were identified 471 from WormBase ParaSite ${ }^{90}$.

\section{Random Forest (RF)}

473 A single-cell dataset published for Schmidtea mediterranea comprising 21,610 cells generated 474 using a droplet-based platform ${ }^{31}$ was employed for this analysis. The relevant files were 475 downloaded from https://shiny.mdc-berlin.de/psca/ including the Schmidtea mediterranea 476 single-cell data comprising 21,610 cells. The Seurat package (version 2.3.4) was used for all 477 analysis of the Schmidtea dataset (https://satijalab.org/seurat/). We only kept cells that 478 expressed at least 200 genes, in a minimum of 3 cells. After QC, 21,612 cells and 28,030 479 transcripts remained. We normalised using NormalizeData function from the Seurat 480 (http://satijalab.org/seurat/). Following normalisation, we identified highly variable genes 481 using the Seurat FindVariableGenes function using the cut-offs stated in the website: $z=0.5$ and 482 mean expression in the range 0.0125 to 3.

483 We identified 22 clusters using the FindClusters function from Seurat with a resolution of 0.6. 484 We chose this resolution to capture most of the clusters with biological variability whilst 485 avoiding overclustering. To annotate each cluster, we used the annotation provided by Plass $e t$ 486 al, $2018^{31}$. 
We accessed the transcriptome reference (version 6) for the asexual strain of Schmidtea mediterranea from planmine ${ }^{91}$. This version is a Trinity de novo transcript assembly ${ }^{92}$. We used orthoMCL ${ }^{93}$ to find 1:1 ortholog genes between $S$. mediterranea and S. mansoni by: (i) collapsing Smp and dd_Smed genes to their root names and choosing clusters with a single Schmidtea and Schistosoma gene; and (ii) removing haplotype Smp genes where doing so would reduce a multiple Smp set to a single Smp; (iii) If a single Smp (after all the above checks) contained multiple Schmidtea genes, we randomly selected one of the Schmidtea genes only if it did not map to more than one orthologue cluster. This gave us a set of SchmidteaSchistosoma orthologous gene-pairs. All Schistosoma genes were then replaced in the Schistosoma single-cell matrix with their $S$. mediterranea orthologs.

We first evaluated the RF classifier on the Schmidtea dataset. We used R package randomForest (version 4.6-14) to train the training set using 500 trees. The RF is a supervised learning method that builds decision trees, trained with a defined set of features (genes). The training set was built using cells from the 22 clusters in the Schmidtea dataset with a maximum of $70 \%$ of cells per cluster. As a first RF test, the training set ( $70 \%$ of cells per cluster) was used to assign a cluster label for the test set (remaining 30\%) of the same dataset. We assigned a class to each cell when a minimum of $16 \%$ of trees in the forest converged onto a decision.

To then use the RF classifier on the Schistosoma data set, a training set was built using cells from the 22 clusters in the Schmidtea dataset with a maximum of $70 \%$ of cells per cluster. This training set was used to assign labels to the Schistosoma mansoni cells using the RF package ${ }^{94}$. The RF decision trees were trained with a defined set of common 692 orthologous genes between $S$. mansoni and $S$. mediterranea.

\section{Conserved Schmidtea-Schistosoma orthologous markers}

511 To identify conserved Schmidtea-Schistosoma one-to-one orthologs, we first identified a high 512 confidence set of one-to-one orthologs. For each S. mansoni predicted protein, we identified 513 the $S$. mediterranea BLASTP ${ }^{95}$ hits, and similarly identified the $S$. mansoni BLASTP hits for 514 each $S$. mediterranea protein. If a S. mansoni gene had haplotypic copies in the S. mansoni V7 515 assembly, we only considered the S. mansoni copy on an assembled chromosome, and

516 discarded the allelic copies of the gene from haplotypic contigs. We considered S. mansoni and 517 S. mediterranea genes to be one-to-one orthologs if they were each other's top BLASTP hits, 
with BLAST $\mathrm{E}<0.05$, and the BLAST E-value of the top BLASTP hit was $1 \mathrm{e}+5$ times lower than the BLAST E-value for the next best hit. This gave us 4764 one-to-one $S$. mansoni-S. mediterranea orthologs. These orthologs were used to find conserved orthologous markers.

521 To identify conserved orthologous markers, we filtered the 4764 1:1 orthologues to retain only 522 those for which both the Schistosoma and Schmidtea genes were identified as Seurat markers with Seurat P-value $\leq 1 \mathrm{e}-30$, using the Seurat Schmidtea clusters from Plass et al $2018^{31}$. If a Schistosoma/Schmidtea gene was in more than one cluster, we only considered the cluster for which it had the lowest (most significant) Seurat P-value.

\section{In situ hybridization (ISH)}

527 Fluorescence in situ hybridization (FISH) and whole-mount colorimetric in situ hybridization 528 (WISH) were performed following previously established protocols ${ }^{14,15,49}$ with modifications specific to schistosomula. Schistosomula were killed with ice-cold 1\% $\mathrm{HCl}$ for $30-60 \mathrm{~s}$ before 530 fixation. Schistosomula were fixed for $\sim 0.5-1$ hour at room temperature in $4 \%$ formaldehyde, $5310.2 \%$ Triton X-100\%, 1\% NP-40 in PBS. Adult parasites were fixed for 4 hours in $4 \%$

532 formaldehyde in PBSTx at room temperature. After fixation, schistosomula and adults were 533 dehydrated in methanol and kept in $-20^{\circ} \mathrm{C}$ until usage. Parasites were rehydrated, permeabilised by $10 \mu \mathrm{g} / \mathrm{mL}$ proteinase $\mathrm{K}$ for $10-20 \mathrm{~min}$ for schistosomula or $20 \mu \mathrm{g} / \mathrm{mL}$ proteinase $\mathrm{K}$ for 30 min for adults, and fixed for 10 mins immediately following proteinase $\mathrm{K}$ treatment.

For hybridization, DIG- riboprobes were used for single FISH and WISH, and FITCriboprobes were used for double FISH. Anti-DIG-POD and anti-FITC-POD antibodies were used for FISH at 1:500-1:1000, and anti-DIG-AP antibody was used for WISH. Anti-DIGPOD and anti-DIG-AP antibodies were incubated overnight at $4{ }^{\circ} \mathrm{C}$ and anti-FITC-POD was incubated for $\sim 4$ hours at room temperature before overnight incubation at $4^{\circ} \mathrm{C}$. For FISH, 2-3 independent experiments were performed, and 5-10 worms were analysed for each experiment. For adult FISH and WISH, two independent experiments were performed, with each experiment containing $\sim 5$ male and $\sim 5$ females. Primers used for cloning a fragment of marker genes and riboprobe generation are listed in Table S7.

\section{Immunostaining and labeling}

546 Anti-acetylated a-tubulin antibody (6-11B-1, Santa Cruz) was incubated at 1:500 in blocking 547 solution (5\% Horse serum, 0.5\% Roche Western Blocking Reagent in TNTx). Secondary 
antibody (anti-mouse Alexa Fluor 633, Invitrogen) was used at 1:250-1:500 and was incubated overnight at $4^{\circ} \mathrm{C}$. For lectin labeling, fluorescein succinylated wheat germ agglutinin (sWGA)

550 (Vector Labs) was used at 1:500 dilution in a blocking solution overnight at $4^{\circ} \mathrm{C}$. Fluorescent dextran was used to label tegument cells ${ }^{6}$. Briefly, schistosomula were transferred to $20 \mu \mathrm{m}$ mesh in order to flush out as much media while retaining parasites inside the mesh. $2.5 \mathrm{mg} / \mathrm{ml}$ dextran biotin-TAMRA-dextran (ThermoFisher Scientific, D3312) was added to the mesh and parasites transferred into a $1.7 \mathrm{ml}$ tube. Immediately after the transfer, schistosomula were vortexed for $\sim 2-4$ minutes at $70 \%$ vortex power, transferred back to $20 \mu \mathrm{m}$ mesh and flushed with schistosomula fixative (4\% formaldehyde, $0.2 \%$ Triton X-100\%, 1\% NP-40 in PBS) before fixing.

\section{Imaging and image processing}

Schistosomula FISH images were taken using an Andor Spinning Disk WDb system (Andor

560 Technology). Adult FISH images were taken using a Zeiss LSM 880 with Airyscan (Carl Zeiss) confocal microscope. Colorimetric WISH images were taken using AxioZoom.V16 (Carl Zeiss). Imaris 9.2 (Bitplane) and Photoshop (Adobe Systems) was used to process acquired images of maximum intensity projections (of z-stacks) and single confocal sections for linear adjustment of brightness and contrast.

\section{Calculating cell numbers in schistosomula}

566 Cercariae and parasites at 0,24 and $48 \mathrm{hr}$ post-transformation were fixed in $5 \%(\mathrm{v} / \mathrm{v})$ formaldehyde 4\% (w/v) sucrose in PBS for $15 \mathrm{~min}$ (throughout staining worms were in $1.5 \mathrm{ml}$ microfuge tubes and spun down $2 \mathrm{~min} 500 \mathrm{G}$ when exchanging solutions). The parasites were then permeabilised in 10\% (w/v) sucrose, $0.5 \%$ Triton-X $100(\mathrm{v} / \mathrm{v})$ for $10 \mathrm{~min}$. Parasites were either stored at $4{ }^{\circ} \mathrm{C}$ in $2 \%$ formaldehyde in PBS, or stained immediately. Staining was in low light level conditions to minimise photobleaching. $1 \mu \mathrm{g} / \mathrm{ml}$ DAPI in PBS was added for $10 \mathrm{~min}$, then parasites were post-fixed in 10\% formaldehdye in PBS for $2 \mathrm{~min}$, washed in $1 \mathrm{X}$ PBS, then resuspended in $0.4 \mathrm{X}$ PBS in $\mathrm{ddH}_{2} \mathrm{O}$ (to discourage salt crystals). $10 \mu \mathrm{l}$ parasites were pipetted onto a glass slide and excess liquid drawn away with whatman filter paper. $10 \mu 1$ ProLong Gold antifade mountant was added to the sample and a glass coverslip dropped over gently. Slides were left at room temperature overnight to set before imaging. A Zeiss LSM 510 Meta confocal microscope was used in conjunction with the Zen software to take a series of $Z$ stacks, imaging 3 individual worms from each timepoint. 
$579 \mathrm{Z}$ stack images were imported into ImageJ software (Import>image sequence) then converted

580 to RGB and split by color (Color>split channels) and the blue channel used for further

581 processing. Using the metadata associated with the file the scale properties were adjusted. The

582 image was cropped if necessary to show only one parasite. The threshold was set to remove

583 any background. The signal above threshold was measured for the whole image stack (image

584 can be inverted and converted to 8 bit for this purpose). The ROI manager was used to measure

585 individual cell nuclei throughout the $\mathrm{Z}$ stack by drawing around the cell on each image of the

586 stack where present. This was imported to the threshold filtered stack and the area measured.

58710 nuclei that were clearly defined and of diverse location and size were measured for each

588 worm to obtain an average nuclei size and signal. In all cases, $\mathrm{Z}$ was used as well as $\mathrm{X}$ and $\mathrm{Y}$

589 to account for the full volume of the nuclei. The total volume for above threshold signal in the

590 worm was divided by the average nuclei size to obtain an estimate for cell number. 


\section{Acknowledgements}

594 The work at WSI was supported by Wellcome (award numbers 206194 and 107475/Z/15/Z).

595 PAN is an investigator of the Howard Hughes Medical Institute. B. glabrata snails used in the

596 United States were provided by the NIAID Schistosomiasis Resource Center of the Biomedical

597 Research Institute (Rockville, MD) through NIH-NIAID Contract HHSN272201700014I for

598 distribution through BEI Resources. We thank the Sequencing and Informatics core facilities

599 at WSI for their contribution. We also thank the following: Gal Horesh for initial technical

600 assistance optimising the dissociation conditions; Catherine McCarthy and Simon Clare for

601 technical support with animal infections and maintenance of the $S$. mansoni life cycle; David

602 Goulding and Claire Cormie at the Electron and Advanced Light Microscopy facility; Jennie

603 Graham and Sam Thompson at the Cytometry Core Facility; Nancy Holroyd, Mandy Sanders,

604 Elizabeth Cook and Nathalie Smerdon for facilitating the submission of 10X samples; and

605 Matthew Jones for 10X training and library preparations. The authors thank Dr. Shristi Pandey

606 for sharing the random forest code used in this work and Dr. Mireya Plass for sharing the 607 planaria dataset.

608

609 Competing financial interests

610 H.M. Bennett is currently employed at Berkeley Lights Inc. which makes commercially 611 available single-cell technology

612

613 


\section{References}

6161 Hoffmann, K. F., Brindley, P. J. \& Berriman, M. Halting harmful helminths.

617 doi:10.1126/science.1261139(2014).

6182 Dorsey, C. H., Cousin, C. E., Lewis, F. A. \& Stirewalt, M. A. Ultrastructure of the

619 Schistosoma mansoni cercaria. Micron 33, 279-323, doi:Pii S0968-4328(01)00019-1

6203 Cioli, D., Pica-Mattoccia, L., Basso, A. \& Guidi, A. Schistosomiasis control:

621 praziquantel forever? Molecular and biochemical parasitology 195, 23-29, 622 doi:10.1016/j.molbiopara.2014.06.002 (2014).

6234 Hockley, D. J. \& McLaren, D. J. Schistosoma mansoni: changes in the outer membrane 624 of the tegument during development from cercaria to adult worm. International journal for 625 parasitology 3, 13-25 (1973).

6265 Collins, J. J., Wendt, G. R., Iyer, H. \& Newmark, P. A. in eLife Vol. 5 (2016).

6276 Wendt, G. R. et al. Flatworm-specific transcriptional regulators promote the 628 specification of tegumental progenitors in Schistosoma mansoni. eLife 7, 629 doi:10.7554/eLife.33221 (2018).

6307 Wilson, R. A. The saga of schistosome migration and attrition. Parasitology 136, 1581631 1592, doi:10.1017/s0031182009005708 (2009).

6328 Wilson, R. A. \& Barnes, P. E. The tegument of Schistosoma mansoni: observations on 633 the formation, structure and composition of cytoplasmic inclusions in relation to tegument 634 function. Parasitology 68, 239-258 (1974).

6359 Sulbarán, G. et al. An invertebrate smooth muscle with striated muscle myosin 636 filaments. doi:10.1073/pnas.1513439112 (2015).

63710 Halton, D. W. \& Gustafsson, M. K. S. Functional morphology of the platyhelminth 638 nervous system. Parasitology 113, S47-S72, doi:Doi 10.1017/S0031182000077891 (1996).

63911 Collins, J. J., 3rd, King, R. S., Cogswell, A., Williams, D. L. \& Newmark, P. A. An 640 atlas for Schistosoma mansoni organs and life-cycle stages using cell type-specific markers 641 and confocal microscopy. PLoS neglected tropical diseases 5, e1009, 642 doi:10.1371/journal.pntd.0001009 (2011).

$64312 \mathrm{Lu}, \mathrm{Z}$. et al. Schistosome sex matters: a deep view into gonad-specific and pairing644 dependent transcriptomes reveals a complex gender interplay. Sci Rep 6, 31150, 645 doi:10.1038/srep31150 (2016).

64613 Senft, A. W., Philpott, D. E. \& Pelofsky, A. H. Electron microscope observations of 647 the integument, flame cells, and gut of Schistosoma mansoni. The Journal of parasitology 47, $648 \quad 217-229(1961)$.

64914 Wang, B., Collins, J. J., 3rd \& Newmark, P. A. Functional genomic characterization of 650 neoblast-like stem cells in larval Schistosoma mansoni. eLife 2, e00768, 651 doi:10.7554/eLife.00768 (2013). 
65215 Wang, B. et al. Stem cell heterogeneity drives the parasitic life cycle of Schistosoma

653 mansoni. eLife 7, doi:10.7554/eLife.35449 (2018).

65416 Hoffmann, K. F., Johnston, D. A. \& Dunne, D. W. Identification of Schistosoma 655 mansoni gender-associated gene transcripts by cDNA microarray profiling. Genome Biology 3 656 (2002).

65717 Fitzpatrick, J. M. et al. An oligonucleotide microarray for transcriptome analysis of 658 Schistosoma mansoni and its application/use to investigate gender-associated gene expression. 659 Molecular and biochemical parasitology 141, 1-13, doi:10.1016/j.molbiopara.2005.01.007 660 (2005).

66118 Chai, M. et al. Transcriptome profiling of lung schistosomula,in vitro cultured 662 schistosomula and adult Schistosoma japonicum. Cellular and molecular life sciences : CMLS 663 63, 919-929, doi:10.1007/s00018-005-5578-1 (2006).

66419 Dillon, G. P. et al. Microarray analysis identifies genes preferentially expressed in the 665 lung schistosomulum of Schistosoma mansoni. International journal for parasitology 36, 1-8, 666 doi:10.1016/j.ijpara.2005.10.008 (2006).

66720 Gobert, G. N. et al. Tissue specific profiling of females of Schistosoma japonicum by 668 integrated laser microdissection microscopy and microarray analysis. PLoS neglected tropical 669 diseases 3, e469, doi:10.1371/journal.pntd.0000469 (2009).

67021 Parker-Manuel, S. J., Ivens, A. C., Dillon, G. P. \& Wilson, R. A. Gene expression 671 patterns in larval Schistosoma mansoni associated with infection of the mammalian host. PLoS 672 neglected tropical diseases 5, e1274, doi:10.1371/journal.pntd.0001274 (2011).

67322 Protasio, A. V., Dunne, D. W. \& Berriman, M. Comparative study of transcriptome 674 profiles of mechanical- and skin-transformed Schistosoma mansoni schistosomula. PLoS 675 neglected tropical diseases 7, e2091, doi:10.1371/journal.pntd.0002091 (2013).

67623 Anderson, L. et al. Schistosoma mansoni Egg, Adult Male and Female Comparative 677 Gene Expression Analysis and Identification of Novel Genes by RNA-Seq. PLoS neglected 678 tropical diseases 9, e0004334, doi:10.1371/journal.pntd.0004334 (2015).

67924 Gobert, G. N., Moertel, L., Brindley, P. J. \& McManus, D. P. Developmental gene 680 expression profiles of the human pathogen Schistosoma japonicum. BMC genomics 10, 681

68225 Ramsköld, D. et al. Full-Length mRNA-Seq from single cell levels of RNA and 683 individual circulating tumor cells. Nat Biotechnol 30, 777-782, doi:10.1038/nbt.2282 (2012).

68426 Pollen, A. A. et al. Low-coverage single-cell mRNA sequencing reveals cellular 685 heterogeneity and activated signaling pathways in developing cerebral cortex. Nature 686 Biotechnology 32, 1053, doi:doi:10.1038/nbt.2967 (2014).

68727 Zeisel, A. et al. Brain structure. Cell types in the mouse cortex and hippocampus 688 revealed by single-cell RNA-seq. Science (New York, N.Y.) 347, 1138-1142, 689 doi:10.1126/science.aaa1934 (2015). 
69028 Karaiskos, N. et al. The Drosophila embryo at single-cell transcriptome resolution.

691 Science (New York, N.Y.) 358, 194-199, doi:10.1126/science.aan3235 (2017).

69229 Cao, J. et al. Comprehensive single-cell transcriptional profiling of a multicellular 693 organism. Science (New York, N.Y.) 357, 661-667, doi:10.1126/science.aam8940 (2017).

69430 Zheng, G. X. Y. et al. Massively parallel digital transcriptional profiling of single cells.

695 Nature Communications 8, 14049, doi:doi:10.1038/ncomms14049 (2017).

69631 Plass, M. et al. Cell type atlas and lineage tree of a whole complex animal by single-

697 cell transcriptomics. Science (New York, N.Y.) 360, doi:10.1126/science.aaq1723 (2018).

69832 Fincher, C. T., Wurtzel, O., de Hoog, T., Kravarik, K. M. \& Reddien, P. W. Cell type 699 transcriptome atlas for the planarian Schmidtea mediterranea. Science (New York, N.Y.) 360, 700 doi:10.1126/science.aaq1736 (2018).

70133 Trapnell, C. et al. The dynamics and regulators of cell fate decisions are revealed by 702 pseudotemporal ordering of single cells. Nat Biotechnol 32, 381-386, doi:10.1038/nbt.2859 703 (2014).

70434 Manno, G. L. et al. RNA velocity of single cells. Nature 560, 494, 705 doi:doi:10.1038/s41586-018-0414-6 (2018).

70635 Reid, A. J. et al. Single-cell RNA-seq reveals hidden transcriptional variation in malaria 707 parasites. doi:doi:10.7554/eLife.33105 (2018).

70836 Wagner, D. E. et al. Single-cell mapping of gene expression landscapes and lineage in 709 the zebrafish embryo. Science (New York, N.Y.) 360, 981-987, doi:10.1126/science.aar4362 710 (2018).

71137 Farrell, J. A. et al. Single-cell reconstruction of developmental trajectories during 712 zebrafish embryogenesis. Science (New York, N.Y.) 360, doi:10.1126/science.aar3131 (2018).

71338 Briggs, J. A. et al. The dynamics of gene expression in vertebrate embryogenesis at 714 single-cell resolution. Science (New York, N.Y.) 360, doi:10.1126/science.aar5780 (2018).

71539 Zeng, A. et al. Prospectively Isolated Tetraspanin(+) Neoblasts Are Adult Pluripotent 716 Stem Cells Underlying Planaria Regeneration. Cell 173, 1593-1608 e1520, 717 doi:10.1016/j.cell.2018.05.006 (2018).

$71840 \quad$ Sanchez Alvarado, A. \& Newmark, P. A. The use of planarians to dissect the molecular 719 basis of metazoan regeneration. Wound Repair Regen 6, 413-420 (1998).

$72041 \quad$ Kiselev, V. Y. et al. SC3: consensus clustering of single-cell RNA-seq data. Nature 721 Methods 14, 483, doi:doi:10.1038/nmeth.4236 (2017).

72242 Butler, A., Hoffman, P., Smibert, P., Papalexi, E. \& Satija, R. Integrating single-cell 723 transcriptomic data across different conditions, technologies, and species. Nat Biotechnol 36, 724 411-420, doi:10.1038/nbt.4096 (2018).

72543 Becht, E. et al. Dimensionality reduction for visualizing single-cell data using UMAP. 726 Nature Biotechnology 37, 38-+, doi:10.1038/nbt.4314 (2019). 
44 Francis, P. \& Bickle, Q. Cloning of a 21.7-Kda Vaccine-Dominant Antigen Gene of Schistosoma-Mansoni Reveals an Ef Hand-Like Motif. Molecular and biochemical parasitology 50, 215-224, doi:Doi 10.1016/0166-6851(92)90218-9 (1992).

73045 Tararam, C. A., Farias, L. P., Wilson, R. A. \& Leite, L. C. Schistosoma mansoni Annexin 2: molecular characterization and immunolocalization. Experimental parasitology 126, 146-155, doi:10.1016/j.exppara.2010.04.008 (2010).

46 Fitzsimmons, C. M. et al. The Schistosoma mansoni tegumental-allergen-like (TAL) protein family: influence of developmental expression on human $\operatorname{IgE}$ responses. PLoS neglected tropical diseases 6, e1593, doi:10.1371/journal.pntd.0001593 (2012).

47 Chen, J. et al. Molecular cloning and expression profiles of Argonaute proteins in Schistosoma japonicum. Parasitology research 107, 889-899, doi:10.1007/s00436-010-1946$7383(2010)$.

73948 Anderson, L., Pierce, R. J. \& Verjovski-Almeida, S. Schistosoma mansoni histones: 740 From transcription to chromatin regulation; an in silico analysis. Molecular and biochemical parasitology 183, 105-114, doi:10.1016/j.molbiopara.2012.03.001 (2012).

49 Collins, J. J., 3rd et al. Adult somatic stem cells in the human parasite Schistosoma mansoni. Nature 494, 476-479, doi:10.1038/nature11924 (2013).

74450 Olson, E. N. MyoD family: a paradigm for development? Genes \& development 4, 745 1454-1461 (1990).

$74651 \quad$ Arber, S., Halder, G. \& Caroni, P. Muscle LIM protein, a novel essential regulator of myogenesis, promotes myogenic differentiation. Cell 79, 221-231 (1994).

74852 Gomes, A. V., Potter, J. D. \& Szczesna-Cordary, D. The role of troponins in muscle contraction. IUBMB Life 54, 323-333, doi:10.1080/15216540216037 (2002).

75053 Laube, B., Hirai, H., Sturgess, M., Betz, H. \& Kuhse, J. Molecular determinants of agonist discrimination by NMDA receptor subunits: analysis of the glutamate binding site on the NR2B subunit. Neuron 18, 493-503 (1997).

75354 Mbikay, M., Seidah, N. G. \& Chrétien, M. Neuroendocrine secretory protein 7B2: 754 structure, expression and functions. The Biochemical journal 357, 329-342 (2001).

75555 SJ, M. Patterns of Gene Expression in Schistosoma mansoni larvae associated with 756 Infection of the Mammalia Host PhD thesis, University of York, (2010).

75756 Lodish, $\mathrm{H}$. et al. Myosin: The Actin Motor Protein. 758 doi:https://www.ncbi.nlm.nih.gov/books/NBK21724/ (2000).

75957 Inomata, H. Scaling of pattern formations and morphogen gradients. Dev Growth Differ 760 59, 41-51, doi:10.1111/dgd.12337 (2017).

76158 Adell, T., Cebria, F. \& Salo, E. Gradients in planarian regeneration and homeostasis. 762 Cold Spring Harb Perspect Biol 2, a000505, doi:10.1101/cshperspect.a000505 (2010). 
59 Braschi, S., Borges, W. C. \& Wilson, R. A. Proteomic analysis of the schistosome tegument and its surface membranes. Memorias do Instituto Oswaldo Cruz 101 Suppl 1, 205212, doi:10.1590/s0074-02762006000900032 (2006).

$60 \mathrm{Li}, \mathrm{X}$. H. et al. The schistosome oesophageal gland: initiator of blood processing. PLoS neglected tropical diseases 7, e2337, doi:10.1371/journal.pntd.0002337 (2013).

768

769

770

771

772

773

774

775

776

777

778

779

780

781

782

783

784

785

786

787

788

789

790

791

792

793

794

795

796

797

798

61 Bogitsh, B. J. \& Carter, O. S. Schistosoma mansoni: ultrastructural studies on the esophageal secretory granules. The Journal of parasitology 63, 681-686 (1977).

62 Wilson, R. A. et al. The Schistosome Esophagus Is a 'Hotspot' for Microexon and Lysosomal Hydrolase Gene Expression: Implications for Blood Processing. PLoS neglected tropical diseases 9, e0004272, doi:10.1371/journal.pntd.0004272 (2015).

63 Mousavi, S. A., Malerod, L., Berg, T. \& Kjeken, R. Clathrin-dependent endocytosis. The Biochemical journal 377, 1-16, doi:10.1042/bj20031000 (2004).

64 Skelly, P. J. \& Shoemaker, C. B. The Schistosoma mansoni host-interactive tegument forms from vesicle eruptions of a cyton network. Parasitology 122 Pt 1, 67-73 (2001).

65 de la Torre-Escudero, E., Perez-Sanchez, R., Manzano-Roman, R. \& Oleaga, A. In vivo intravascular biotinylation of Schistosoma bovis adult worms and proteomic analysis of tegumental surface proteins. Journal of proteomics 94, 513-526, doi:10.1016/j.jprot.2013.09.020 (2013).

66 McCarthy, E. et al. Leucine aminopeptidase of the human blood flukes, Schistosoma mansoni and Schistosoma japonicum. International journal for parasitology 34, 703-714, doi:10.1016/j.ijpara.2004.01.008 (2004).

67 Collins, J. J., 3rd \& Newmark, P. A. It's no fluke: the planarian as a model for understanding schistosomes. PLoS Pathog 9, e1003396, doi:10.1371/journal.ppat.1003396 (2013).

68 Eriksson, K. S., Maule, A. G., Halton, D. W., Panula, P. A. \& Shaw, C. GABA in the nervous system of parasitic flatworms. Parasitology 110 ( Pt 3), 339-346 (1995).

69 Collins, J. J., 3rd et al. Genome-wide analyses reveal a role for peptide hormones in planarian germline development. PLoS biology 8, e1000509, doi:10.1371/journal.pbio.1000509 (2010).

70 Miller, C. M. \& Newmark, P. A. An insulin-like peptide regulates size and adult stem cells in planarians. Int J Dev Biol 56, 75-82, doi:10.1387/ijdb.113443cm (2012).

71 Pandey, S., Shekhar, K., Regev, A. \& Schier, A. F. Comprehensive Identification and Spatial Mapping of Habenular Neuronal Types Using Single-Cell RNA-Seq. Current biology : $C B$ 28, 1052-1065.e1057, doi:10.1016/j.cub.2018.02.040 (2018).

72 Collins, J. J., 3rd. Platyhelminthes. Current biology : $C B$ 27, R252-R256, doi:10.1016/j.cub.2017.02.016 (2017). 
79973 Wilson, R. A., University of York, Y., UK, Coulson, P. S. \& University of York, Y.,

800 UK. Schistosome vaccines: a critical appraisal. Mem. Inst. Oswaldo Cruz 101, 13-20, 801 doi:10.1590/S0074-02762006000900004 (2006).

80274 Wilson, R. A., Li, X. H. \& Castro-Borges, W. in Parasites \& vectors Vol. 9 (2016).

80375 Roberts-Galbraith, R. H., Brubacher, J. L. \& Newmark, P. A. A functional genomics 804 screen in planarians reveals regulators of whole-brain regeneration. eLife $\mathbf{5}$, 805 doi:10.7554/eLife.17002 (2016).

80676 Taft, A. S. \& Yoshino, T. P. Cloning and functional characterization of two calmodulin 807 genes during larval development in the parasitic flatworm Schistosoma mansoni. The Journal 808 of parasitology $97,72-81$, doi:10.1645/GE-2586.1 (2011).

80977 Katsumata, T., Kohno, S., Yamaguchi, K., Hara, K. \& Aoki, Y. Hatching of 810 Schistosoma mansoni eggs is a $\mathrm{Ca} 2+/$ calmodulin-dependent process. Parasitology research 811 76, 90-91 (1989).

81278 Zhang, S. et al. Quantifying the mechanics of locomotion of the schistosome pathogen 813 with respect to changes in its physical environment. $J R$ Soc Interface 16, 20180675, 814 doi:10.1098/rsif.2018.0675 (2019).

81579 Witchley, J. N., Mayer, M., Wagner, D. E., Owen, J. H. \& Reddien, P. W. Muscle cells 816 provide instructions for planarian regeneration. Cell reports 4, 633-641, 817 doi:10.1016/j.celrep.2013.07.022 (2013).

$81880 \quad$ Davies, E. L. et al. Embryonic origin of adult stem cells required for tissue homeostasis 819 and regeneration. eLife 6, doi:10.7554/eLife.21052 (2017).

82081 WILSON, A. PROTONEPHRIDIA. Biological Reviews - Wiley Online Library, 821 doi:10.1111/j.1469-185X.1974.tb01572.x (1974).

82282 Picard, M. A. et al. Sex-Biased Transcriptome of Schistosoma mansoni: Host-Parasite 823 Interaction, Genetic Determinants and Epigenetic Regulators Are Associated with Sexual 824 Differentiation. PLoS neglected tropical diseases 10, e0004930, 825 doi:10.1371/journal.pntd.0004930(2016).

82683 Mann, V. H., Morales, M. E., Rinaldi, G. \& Brindley, P. J. Culture for genetic 827 manipulation of developmental stages of Schistosoma mansoni. Parasitology 137, 451-462, 828 doi:10.1017/S0031182009991211 (2010).

82984 Peak, E., Chalmers, I. W. \& Hoffmann, K. F. Development and validation of a 830 quantitative, high-throughput, fluorescent-based bioassay to detect schistosoma viability. PLoS 831 neglected tropical diseases 4, e759, doi:10.1371/journal.pntd.0000759 (2010).

83285 McCarthy, D. J., Campbell, K. R., Lun, A. T. \& Wills, Q. F. Scater: pre-processing, 833 quality control, normalization and visualization of single-cell RNA-seq data in R. 834 Bioinformatics (Oxford, England) 33, 1179-1186, doi:10.1093/bioinformatics/btw777 (2017).

$83586 \quad$ SingleCellExperiment: S4 Classes for Single Cell Data. R package version 1.5.2. 836 (Bioconductor, 2019). 
83787 Lun, A. T. L., Bach, K. \& Marioni, J. C. Pooling across cells to normalize single-cell

838 RNA sequencing data with many zero counts. Genome Biology 17, 75,

839 doi:doi:10.1186/s13059-016-0947-7 (2016).

84088 Alexa A, R. J. topGO: Enrichment Analysis for Gene Ontology, 2019).

$84189 \quad$ Szklarczyk, D. et al. STRING v11: protein-protein association networks with increased

842 coverage, supporting functional discovery in genome-wide experimental datasets. Nucleic

843 acids research 47, D607-D613, doi:10.1093/nar/gky1131 (2019).

$84490 \quad$ Howe, K. L., Bolt, B. J., Shafie, M., Kersey, P. \& Berriman, M. WormBase ParaSite 845 a comprehensive resource for helminth genomics. Molecular and biochemical parasitology 846 215, 2-10, doi:10.1016/j.molbiopara.2016.11.005 (2017).

84791 Brandl, H. et al. PlanMine - a mineable resource of planarian biology and biodiversity.

848 Nucleic acids research 44, D764-773, doi:10.1093/nar/gkv1148 (2016).

84992 Haas, B. J. et al. De novo transcript sequence reconstruction from RNA-seq using the 850 Trinity platform for reference generation and analysis. Nat Protoc 8, 1494-1512, 851 doi:10.1038/nprot.2013.084 (2013).

85293 Li, L., Christian J. Stoeckert, J. \& Roos, D. S. OrthoMCL: Identification of Ortholog 853 Groups for Eukaryotic Genomes. doi:10.1101/gr.1224503 (2003).

85494 Breiman, L. Random Forests. Machine Learning 45, 5-32, 855 doi:10.1023/A:1010933404324 (2001).

85695 Altschul, S. F. et al. Gapped BLAST and PSI-BLAST: a new generation of protein 857 database search programs. Nucleic acids research 25, 3389-3402, doi:10.1093/nar/25.17.3389 858 (1997). 


\section{Figure 1}

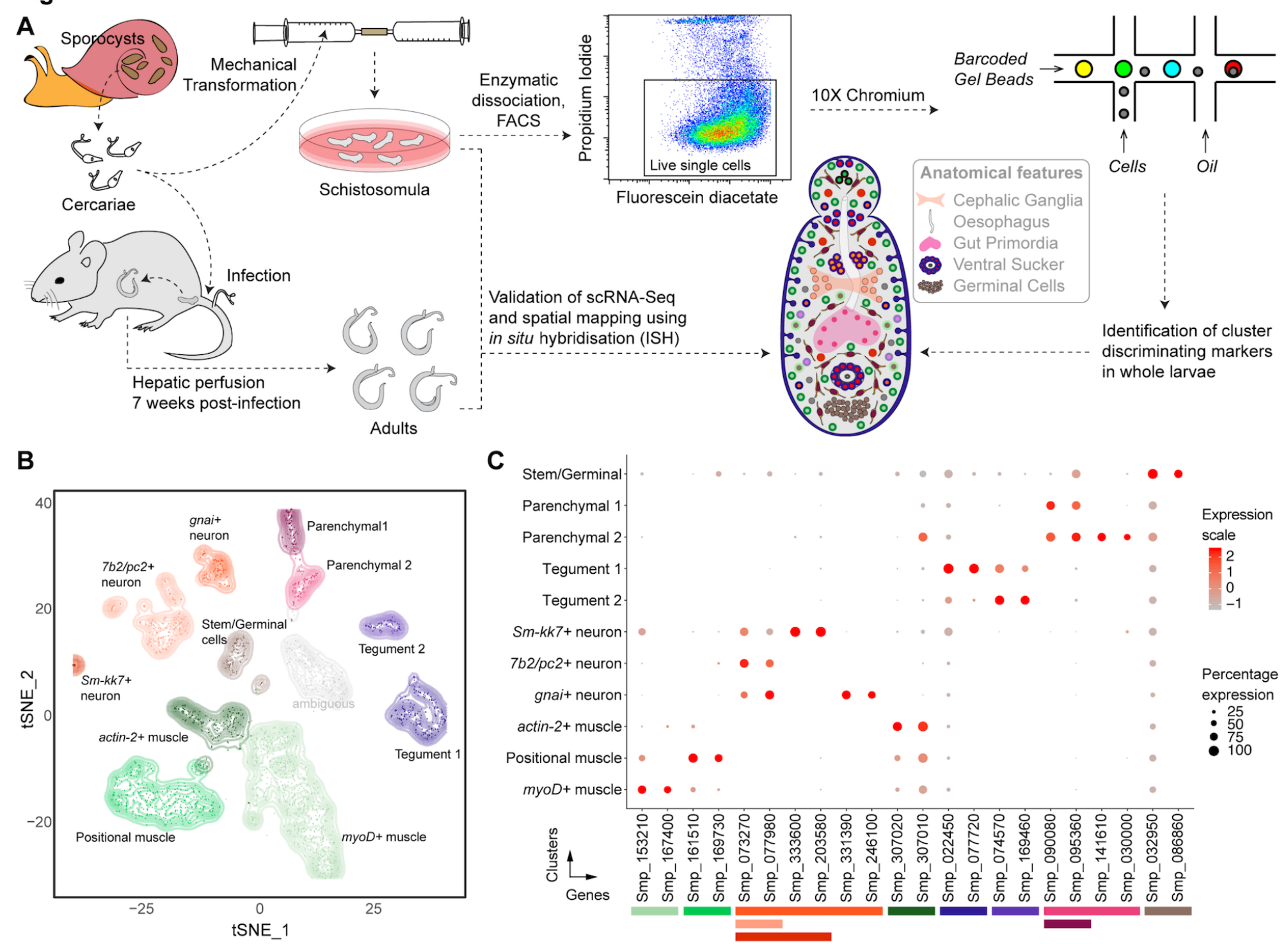

Figure 1. Identification of 11 transcriptionally distinct cell types in schistosomula.

(A) Experimental scheme describing the sources of the parasite material, single-cell analysis and validation pipeline. Approximately 5,000 schistosomula per experiment were dissociated, followed by enrichment of fluorescein diacetate $($ FDA + ) live cells using fluorescence-activated cell sorting (FACS). Cells were loaded according to the 10X Chromium single-cell 3' protocol. Clustering was carried out to identify distinct populations and population-specific markers. Validation of population-specific markers was performed by in situ hybridisation (ISH). (B) t-distributed stochastic neighbour embedding (t-SNE) representation of 2,144 schistosomulum single cells. Clusters are coloured, distinctively labelled, and emphasised with density contours. One ambiguous cluster is de-emphasised and shown in grey. (C) Gene expression profiles of population markers identified for each of the cell clusters. The colours represent the level of expression from dark red (high expression) to light red (low expression). The sizes of the circles represent the percentages of cells in those clusters that expressed a specific gene. The colour bars under gene IDs represent the clusters in (B). 
Figure 2
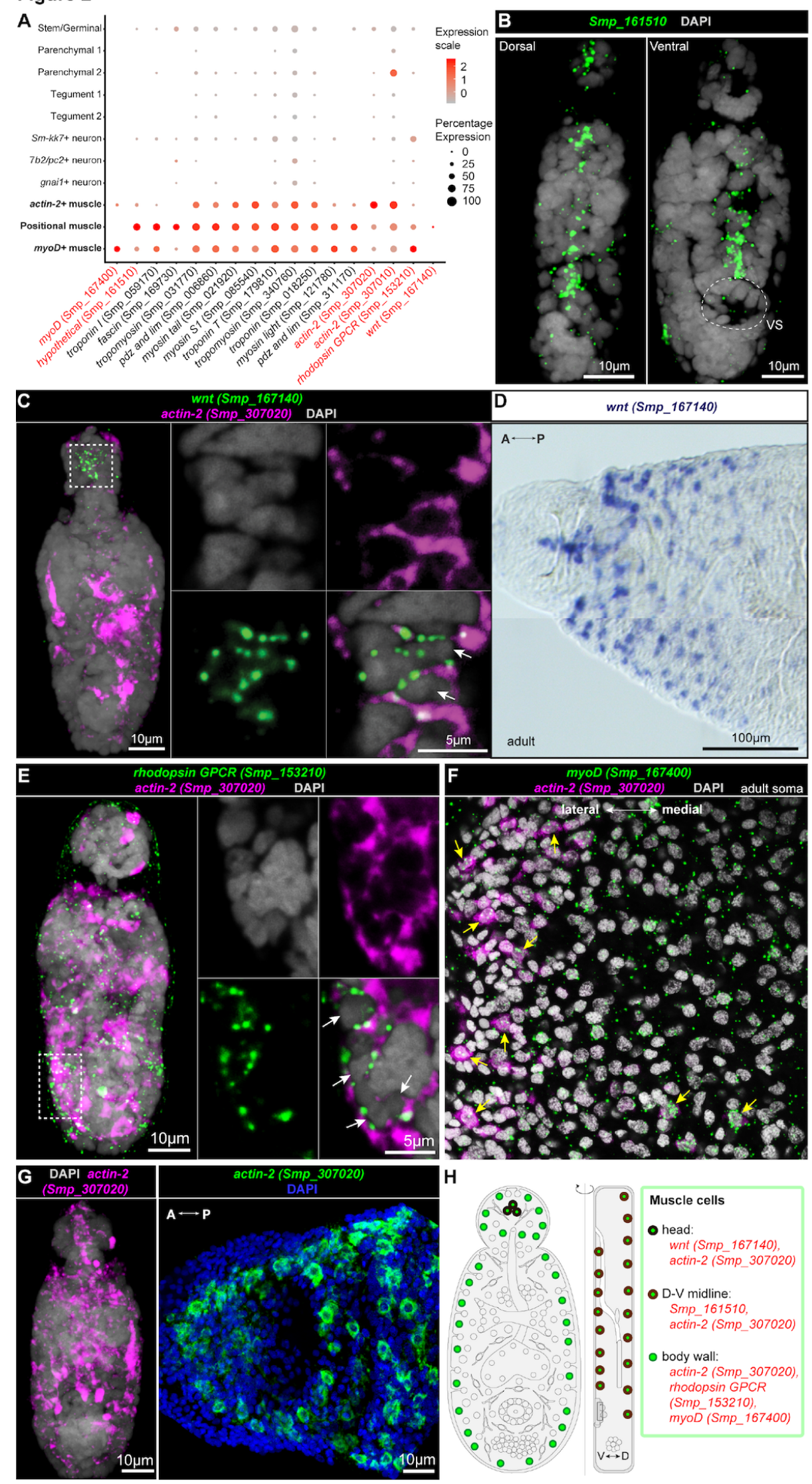

Figure 2. Muscle cells express positional information underlying parasite development. (A) Expression profiles of cell markers that are specific or enriched in the muscle clusters. Genes shown in red were validated by ISH. (B) FISH of Smp_161510. Smp_161510-expressing cells are found in dorsal and ventral sides along the midline. VS: ventral sucker. (C) Double FISH of wnt (Smp_167140) and actin-2 (Smp_307020). wnt is expressed in a subset of actin-2+ cells in the head of the worm (white arrows). (D) Wholemount in situ hybridisation (WISH) of wnt in the head region of the adult worm. A: Anterior; P: Posterior. (E) Double FISH of rhodopsin GPCR (Smp_153210) with actin-2 (Smp_307020). Left: MIP; Right: single magnified confocal sections of the dotted box. White arrows indicate doublepositive cells. (F) Double FISH of myoD (Smp_167400) and actin-2 (Smp_307020) in adult soma. Scattered expression of $m y o D$ throughout the soma, with few double-positive cells (yellow arrows). (G) Spatial distribution of actin-2 (Smp_307020) throughout the body of the parasite. Left panel: schistosomulum; Right panel: adult male. (H) Schematic that summarises the muscle cell types in 2-day schistosomula. Marker genes identified in the current study are indicated in red. All previously reported genes are shown in black. V: Ventral; D: Dorsal. 
Figure 3
A

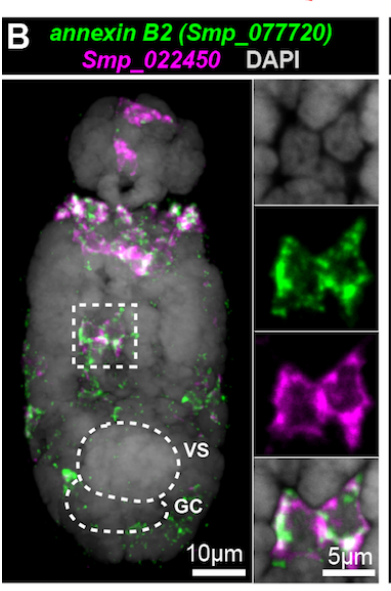

D
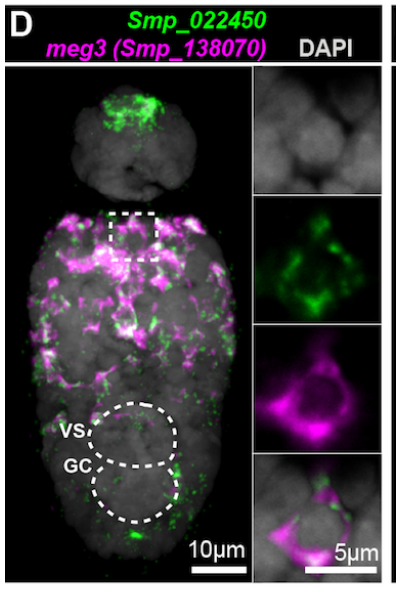
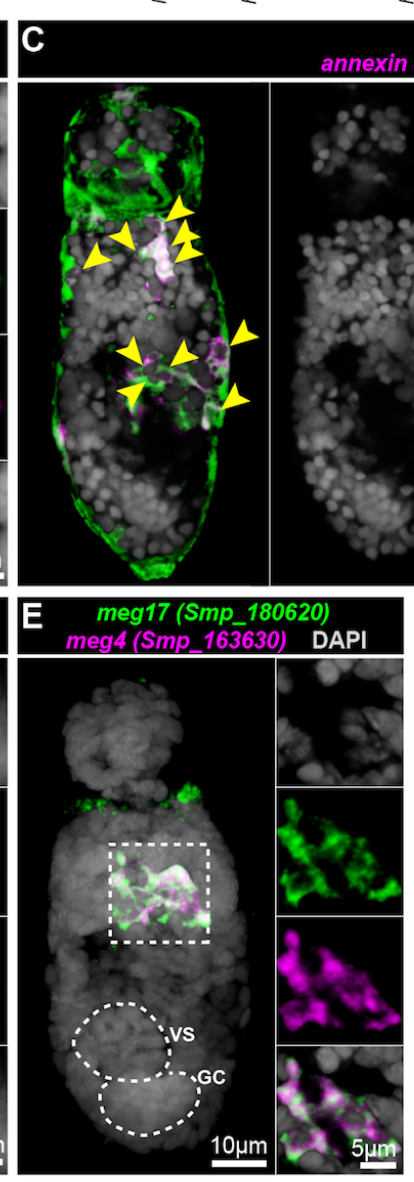

Expression

scale

1

Percentage Expression

$: 20$

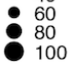

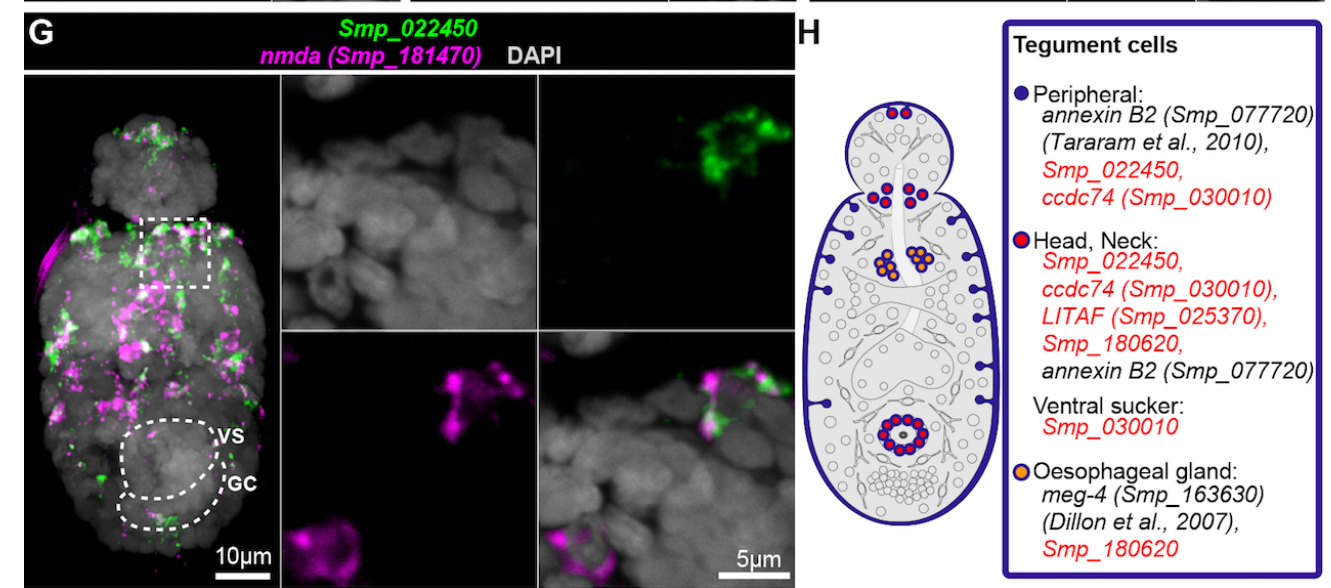

Figure 3. Two distinct populations of tegumental cells in schistosomula.

(A) Expression profiles of cell marker genes that are specific of or enriched in the tegument clusters. Genes validated by ISH are marked in red. (B) Double FISH of Tegument 1 markers annexin B2 (Smp_077720) and Smp_022450. The majority of the cells show co-localisation (white signal). MIP on the left, and zoomedin confocal sections on the right. (C) annexin B2+ cells have taken up the fluorescent dextran. Yellow arrowheads indicate double positive cells. Single confocal sections are shown. (D) Double FISH of Smp_022450 and meg3

(Smp_138070), both Tegument 1 markers. The majority of the cells show co-localisation (white signal). (E) Double FISH of megl 7 (Smp_180620) with a known oesophageal gland gene meg4 (Smp_163630). meg17 is expressed in other regions of the body including in the oesophageal gland. (F-G) Double FISH of Tegument 1 marker (Smp_022450) with (F) $c c d c 74$ (Smp_030010) and (G) nmda (Smp_181470). The majority of cells show co-localisation (white signal), while a subset of cells in the anterior portion of the worm show single positive cells for Tegument 2 markers. (H) Schematic that summarises the tegument cell populations in 2-day schistosomula. Marker genes identified in the current study are indicated in red. All previously reported genes are shown in black.VS: ventral sucker; GC: germinal cell cluster. 
bioRxiv preprint doi: https://doi.org/10.1101/754713; this version posted September 11, 2019. The copyright holder for this preprint (which was not certified by peer review) is the author/funder, who has granted bioRxiv a license to display the preprint in perpetuity. It is made available under aCC-BY-NC-ND 4.0 International license.

\section{Figure 4}

A

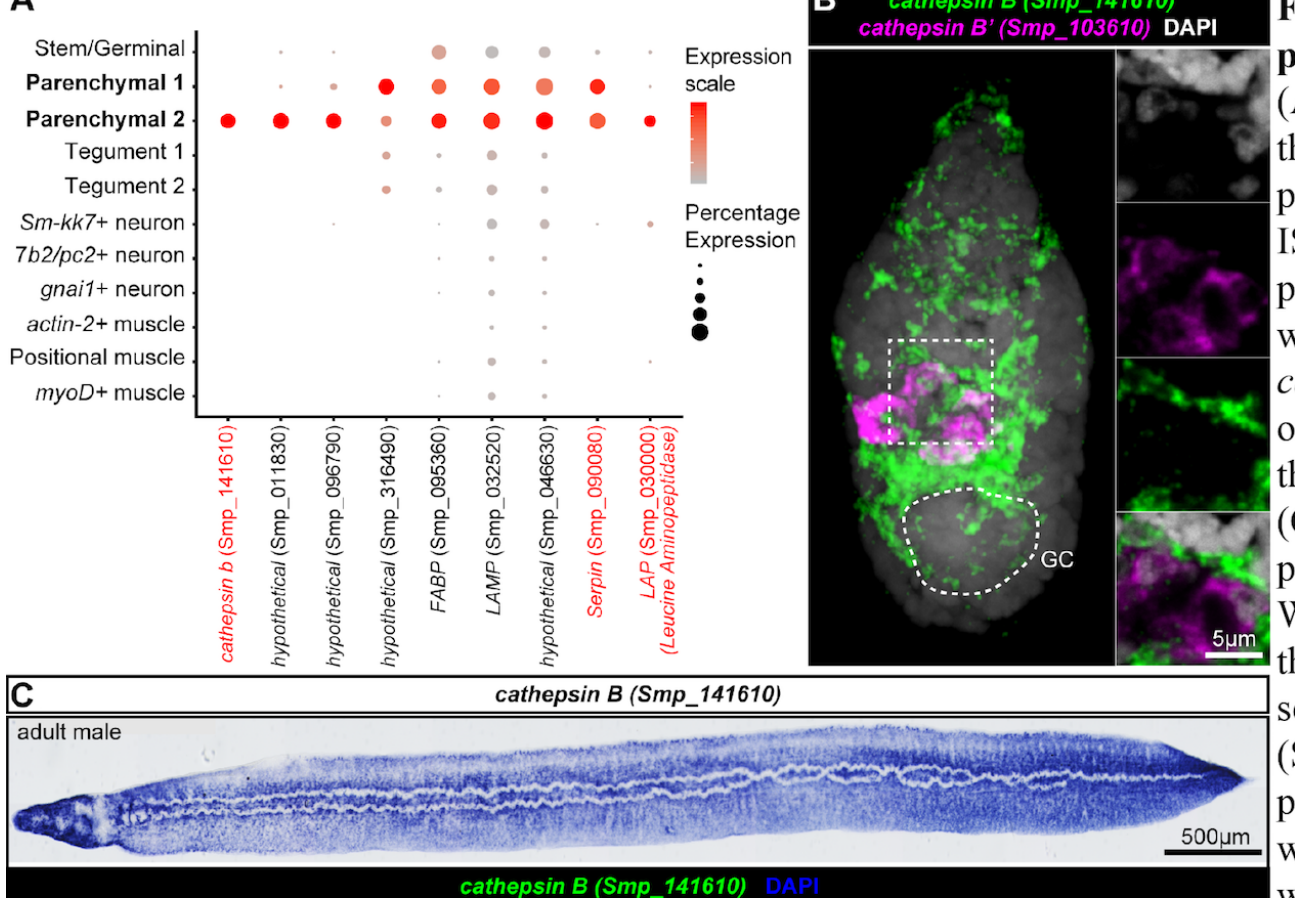

.

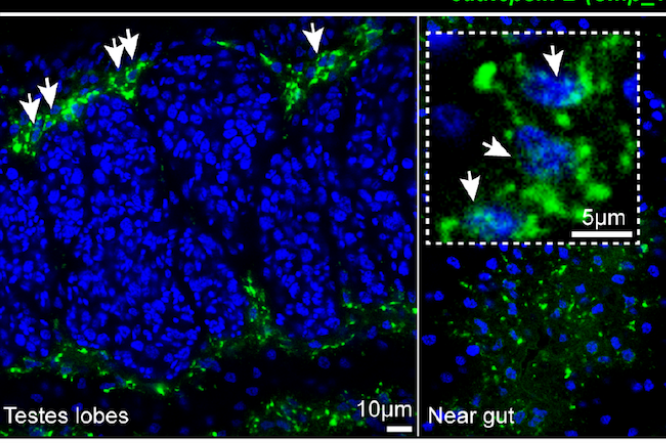

$\bar{D}$

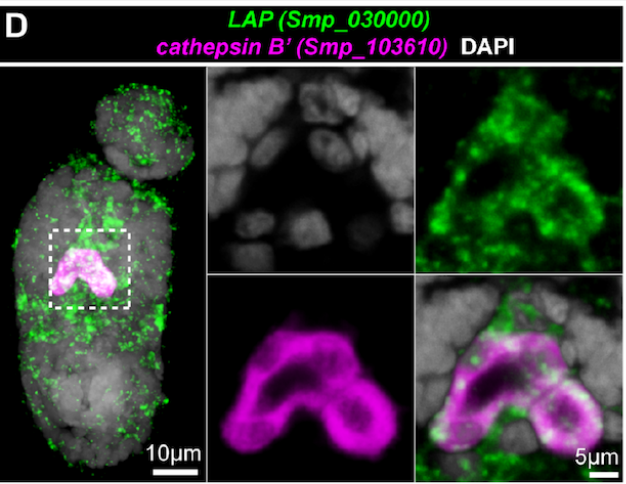

$\mathrm{E}$

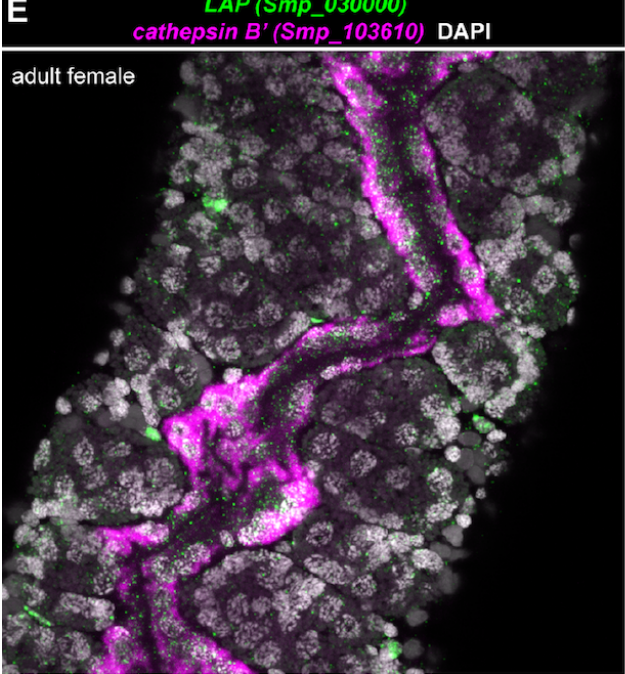

\section{B}

Parenchymal/Gut cells

Parenchymal 1 and 2 :

serpin (Smp_090080)

cathepsin B (Smp 141610),

LAP (Smp_030000) (McCarthy et al., 2004)

- Gut primordia

LAP (Smp 030000)

cathepsin B' (Smp_103610) (Caffrey et al., 2004)
Figure 4. Identification of schistosome parenchymal and primordial gut cells.

(A) Expression profiles of cell marker genes that are specific or enriched in the

parenchymal clusters. Genes validated by ISH are marked in red. (B) Double FISH of parenchymal cathepsin B (Smp_141610) with a known marker of differentiated gut, cathepsin B'(Smp_103610). No expression of parenchymal cathepsin $B$ is observed in the primordial gut. GC: germinal cell cluster. (C) WISH (top) and FISH (bottom) of parenchymal cathepsin $B$ in adult males. White arrowheads indicate positive cells in the bottom part of the figure. Single confocal sections shown for FISH. (D-E) lap (Smp_03000) is expressed in both parenchyma and in the (D) gut primordia as well as (E) adult gut, shown by double FISH with the gut cathepsin B (Smp_103610). (F) Schematic that summarises the parenchymal cell populations in 2-day schistosomula. Marker genes identified in the current study are indicated in red. All previously reported genes are shown in black. 


\section{Figure 5}
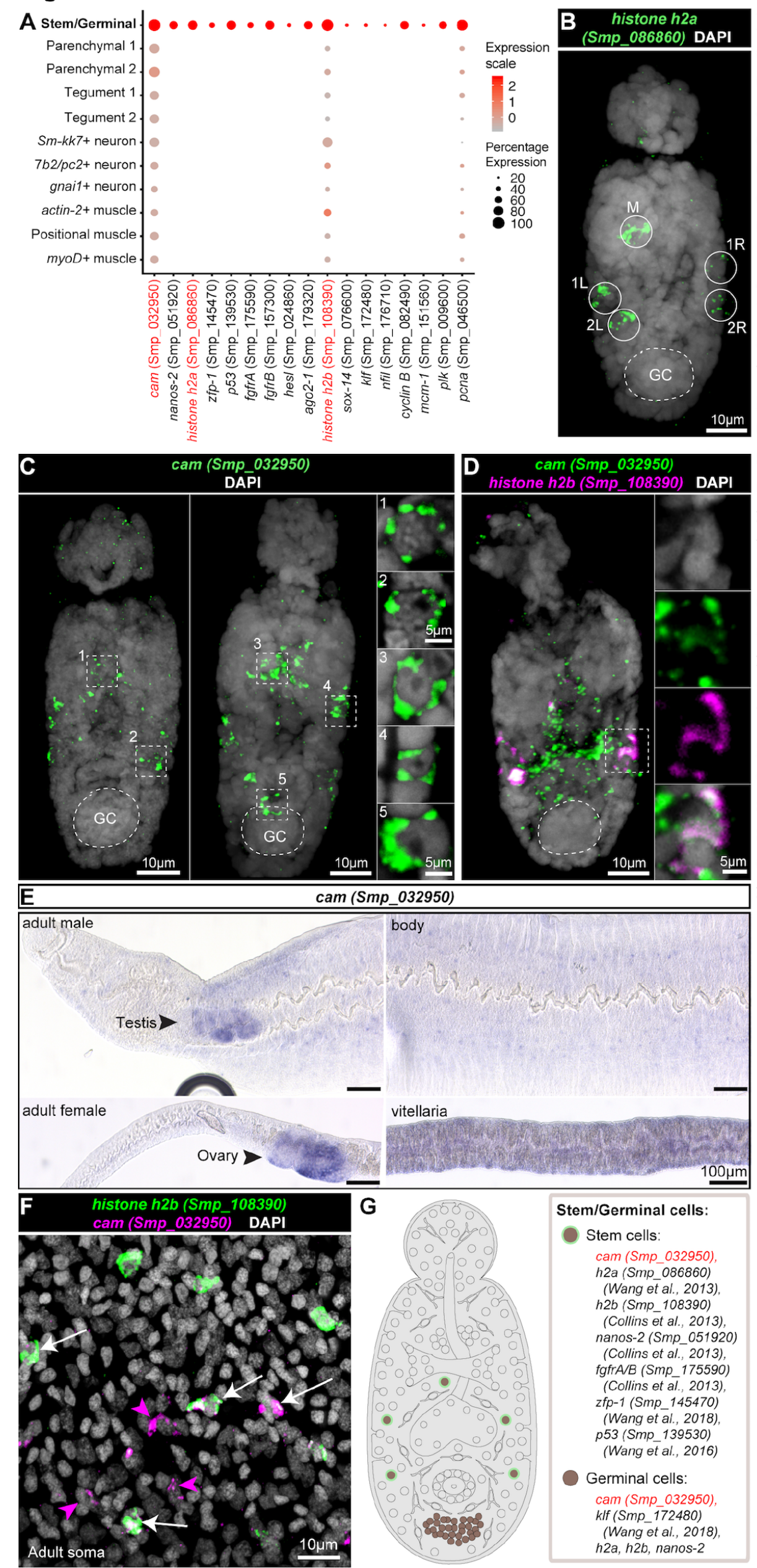

Stem/Germinal cells:

Stem cells:

cam (Smp_032950) h2a (Smp_086860) (Wang et al., 2013), h2b (Smp_108390) (Collins et al, 2013), nanos-2 (Smp_051920) (Collins et al., 2013), fgfrA/B (Smp_175590) (Collins et al., 2013) zfp-1 (Smp_145470) (Wang et al., 2018), 53 (Smp_139530) (Wang et al., 2016)

Germinal cells: cam (Smp_032950) klf (Smp_172480) (Wang et al., 2018) h2a, h2b, nanos-2
Figure 5. A single cluster of stem cells in 2-day old schistosomula.

(A) Expression profiles of cell marker genes that are specific or enriched in the stem/germinal cell cluster. Genes validated by ISH are marked in red. (B) FISH of $h 2 a$ (Smp 086860) shows $\sim 5$ stem cells located at distinct locations - 1 medial cell (M) and 2 lateral cells on each side (1L and $2 \mathrm{~L}$, 1R and 2R; L: left; R: right). (C) FISH of calmodulin (Smp_032950) shows a similar localisation pattern as $h 2 a$, with some worms with a few more cam + cells in the medial region as well as in the germinal cell cluster region. GC: germinal cluster (D) Double FISH of calmodulin (Smp_032950) and a previously validated schistosome stem cell marker $h 2 b$ (Smp_108390). (B-D) MIP is shown for the whole worms, and magnified single confocal sections are shown for the dotted box area. (E) WISH of calmodulin (Smp_032950) in adult parasites shows enriched expression in the gonads including testis, ovary, and vitellarium, as well as in the mid-animal body region. (F) Double FISH of calmodulin and $h 2 b$ in adult soma. A single confocal section is shown. White arrows indicate co-localisation of two genes and magenta arrows indicate expression of only one gene. (G) Schematic that summarises the stem and germinal cell populations in 2-day schistosomula. Marker genes identified in the current study are indicated in red. All previously reported genes are shown in black. 


\section{Figure 6}
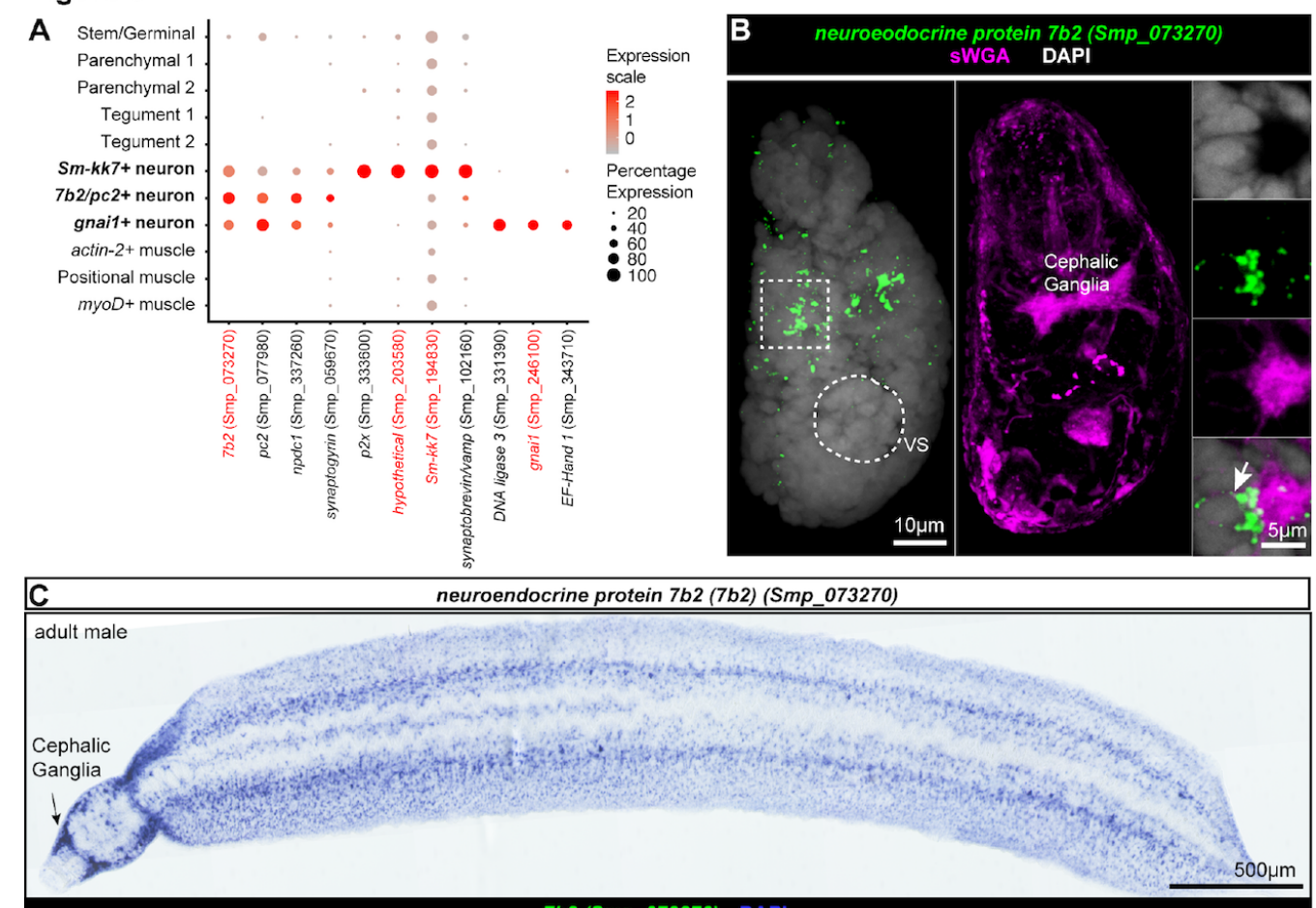

\section{.}

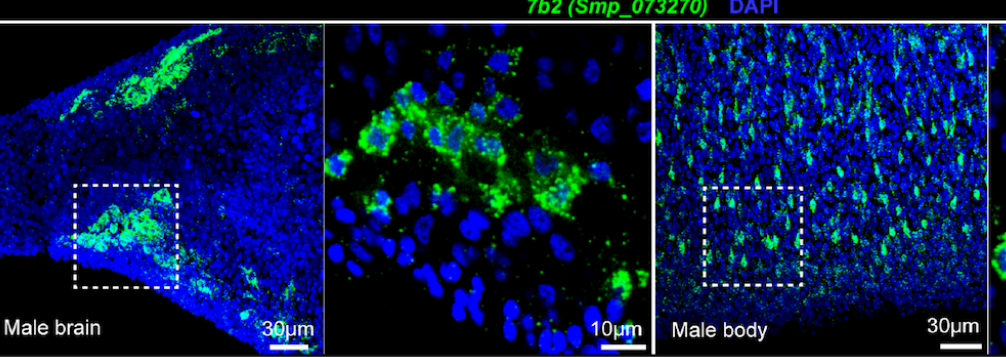

D
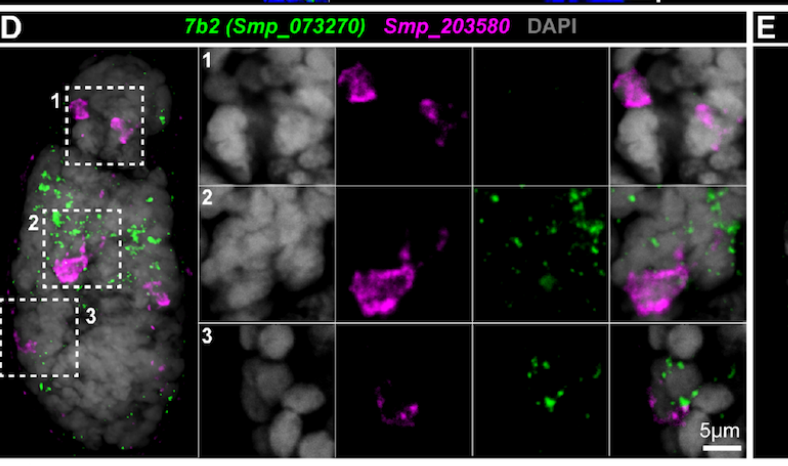

E $\quad$ Sm-kk7 (Smp_194830)

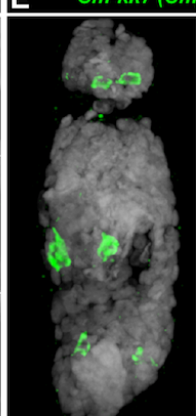

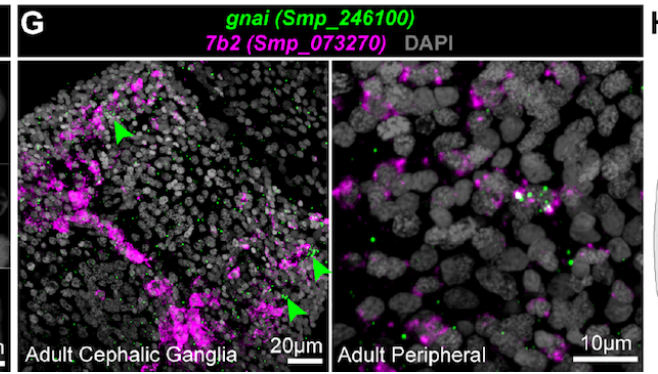

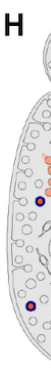

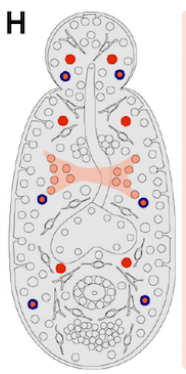

is

6. $10 \mu \mathrm{m}$ oum

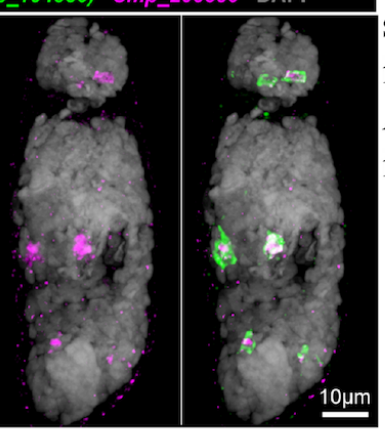

Nervous system

cephalic ganglia: $7 b 2$ (Smp_073270), pc2 (Smp_077980)

peripheral: $S m-k k 7$ (Smp_194830), Smp_203580

peripheral gnia (Smp_246100), $7 b 2$, pc2
Figure 6. Heterogeneity in cells of schistosomula nervous system.

(A) Expression profiles of cell marker genes that are specific or enriched in the neuronal clusters. Genes validated by ISH are marked in red. (B) Cephalic ganglia marked by sWGA lectin shows co-localisation with $7 b 2$ (Smp 073270). White arrows indicate co-localisation of gene- sWGA lectin (C) WISH (top) and FISH (bottom) of $7 b 2$ (Smp_073270) in adults. Single confocal sections are shown for FISH. (D) Double FISH of $7 b 2$ (Smp_073270) and Smp_203580 shows that six cells that are Smp_203580+ (in magenta) do not co-localise with $7 b 2+$ cells (in green). (E) Double FISH of Smp_203580 with Sm-kk7 (Smp_194830). All Smp_203580+ cells co-localise with Sm-kk7. (F) gnai (Smp_246100) FISH shows expression in a few cells in the head and in the body region. (G) Double FISH of gnai with $7 b 2$ shows co-localisation in the peripheral neurons. Single confocal sections are shown. (H) Schematic that summarises the neuronal cell populations in two-day schistosomula. Marker genes identified in the current study are indicated in red. All previously reported genes are shown in black. 
bioRxiv preprint doi: https://doi.org/10.1101/754713; this version posted September 11, 2019. The copyright holder for this preprint (which was not certified by peer review) is the author/funder, who has granted bioRxiv a license to display the preprint in perpetuity. It is made available under aCC-BY-NC-ND 4.0 International license.

\section{Figure 7}

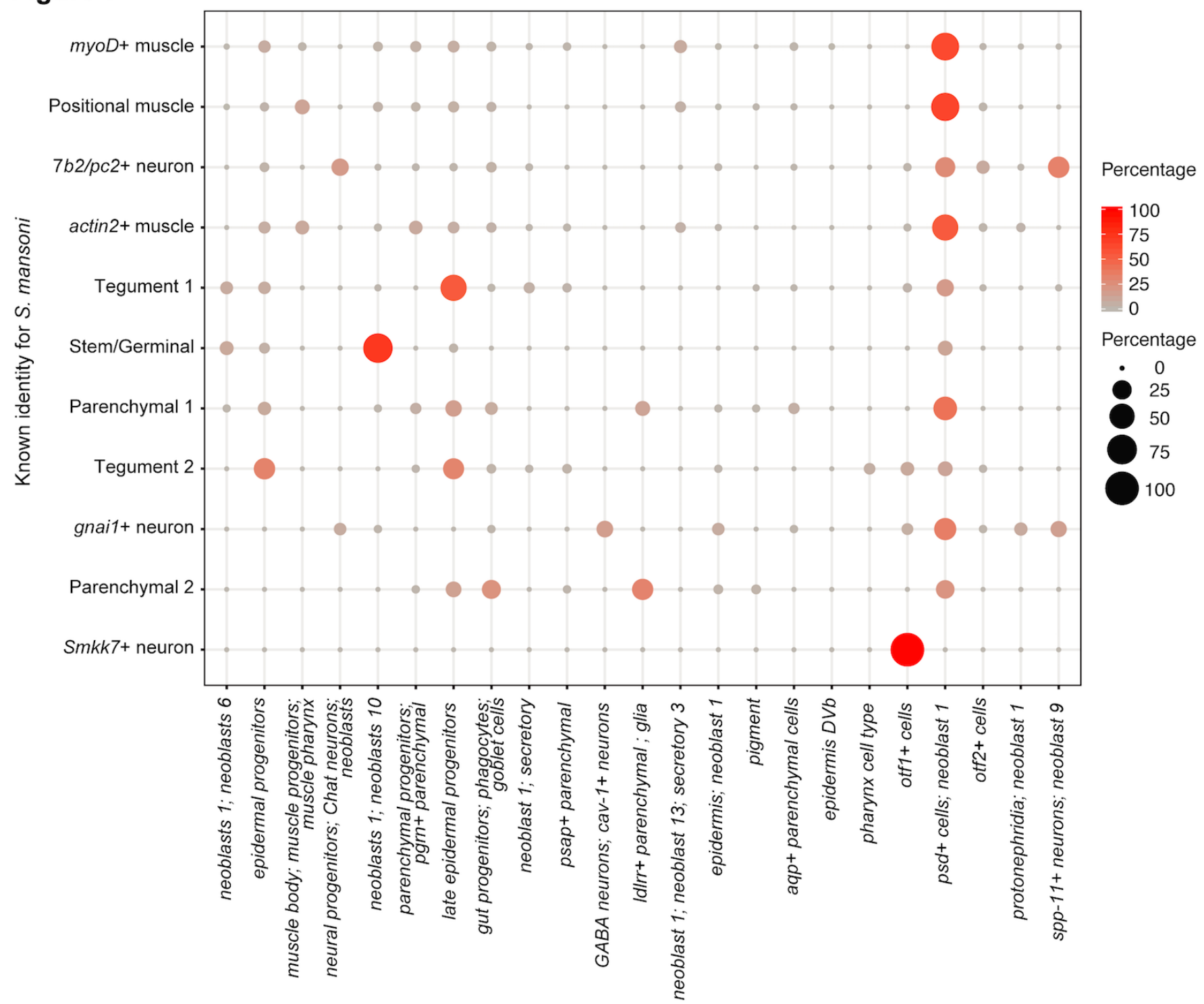

Predicted cell identity from S. mediterranea

Figure 7. Conserve gene expression patterns in stem cells and neurons between $\boldsymbol{S}$. mansoni and $\boldsymbol{S}$ chmidtea mediterranea. Dot plot showing the percentage of cells within each of the schistosomulum clusters (rows) that were mapped to Schmidtea mediterranea scRNA seq dataset ${ }_{31}$ (columns) using a multiclass random forest classifier (RF). The colours and size of the circles represent the proportion of cells assigned to a particular label. Large circles in red represent $100 \%$ of cells. The small circles in light red represent $0 \%$ of cells. 


\section{Supplementary Figure 1}

A

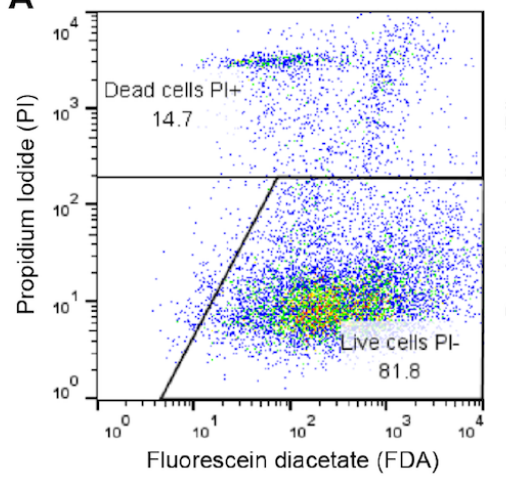

Liberase DL digestion $37^{\circ} \mathrm{C}, 30 \mathrm{mins}$

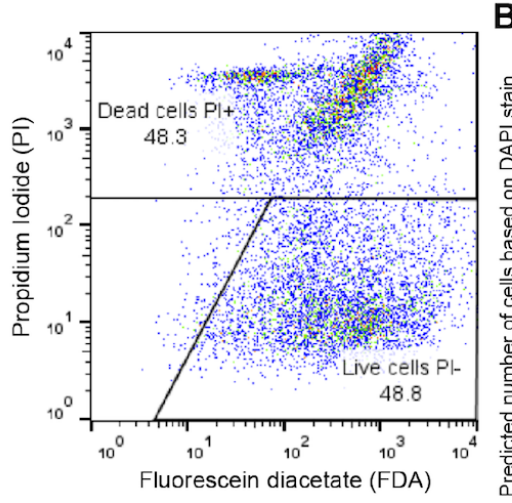

Pronase $0.5 \%$ digestion $37^{\circ} \mathrm{C}, 30 \mathrm{mins}$

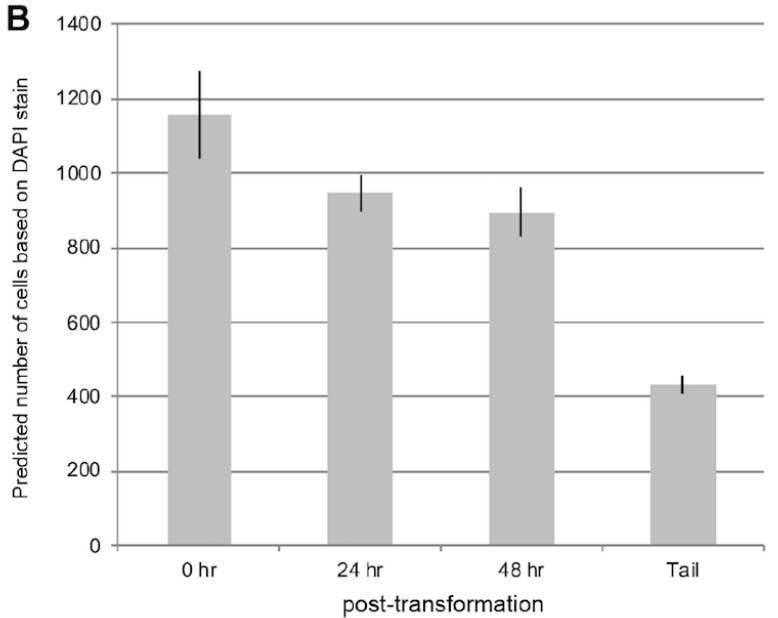

C

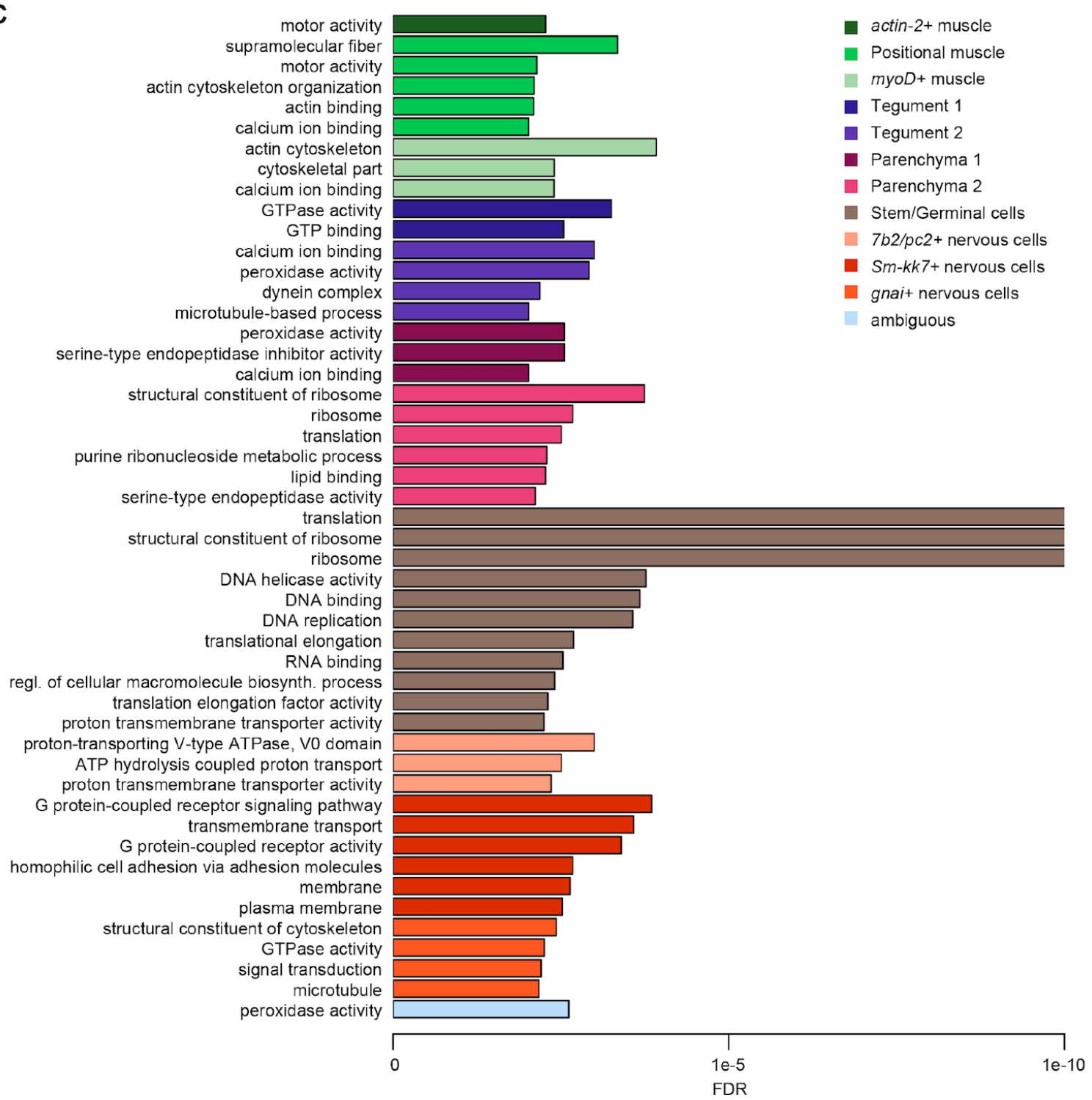

Supplementary Figure 1. (A) Comparison between protocols to dissociate schistosomula. Flow cytometry-based assessment of dissociation with either Liberase DL $(750 \mu 1 / \mathrm{ml})$ (left) or Pronase $0.5 \%$ (right) revealed that the former led to more live cells than the latter. (B) Predicted number of cells that comprises an in vitro-transformed schistosomulum. The bar chart shows the number of cells counted in schistosomula immediately after mechanical transformation ( $0 \mathrm{hr}$, i.e. cercaria head), after one day $(24 \mathrm{hr})$ and two days ( $48 \mathrm{hrs}$ ) in culture. 'Tail' represents the number of cells counted in the tail detached from the cercaria during the mechanical transformation. Mean of number of cells counted in 3 schistosomula per timepoint (C) Significantly enriched GO terms for the marker genes in each cell cluster. Plot showing terms with FDR $<0.01$ from a Fisher's exact test and coloured by cell types. The x-axis indicates - $\log 10 \mathrm{FDR}$ values, where an arbitrary maximum value of 10 was set. 
bioRxiv preprint doi: https://doi.org/10.1101/754713; this version posted September 11, 2019. The copyright holder for this preprint (which was not certified by peer review) is the author/funder, who has granted bioRxiv a license to display the preprint in perpetuity. It is made available under aCC-BY-NC-ND 4.0 International license.

Supplementary Figure 2
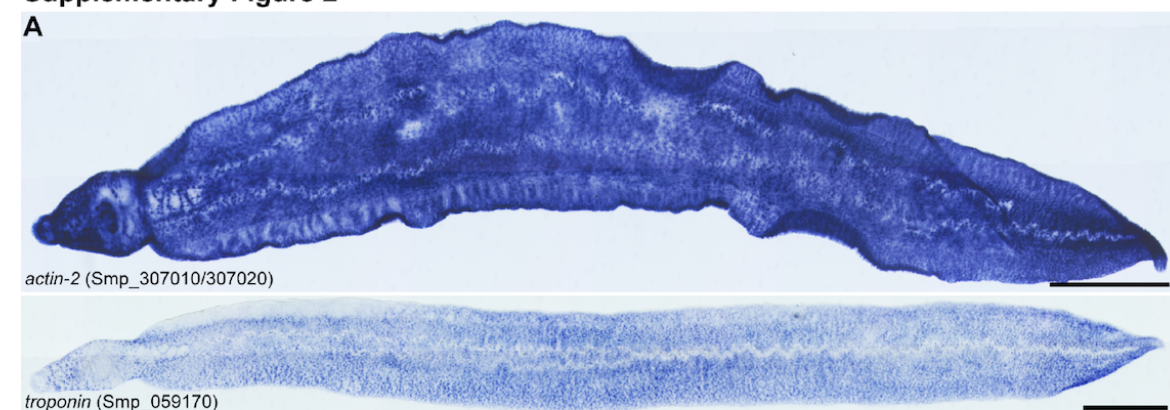

troponin (Smp_059170)

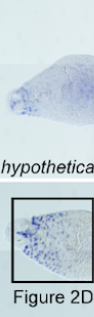

O

wnt (Smp_167140)
Supplementary Figure 2. (A) WISH of indicated markers and signalling molecules enriched in a subset of muscle cells in adult schistosomes. For $w n t$, the boxed region is shown in Figure 2D. On the right, WISH experiment shows that wnt expression is conserved in the anterior end of juvenile parasites collected from mice 3 weeks post-infection. (B) FISH of muscle markers in indicated regions of the adult worms. (C) Double FISH of selected muscle markers. White arrows: double positive cells; green arrowheads: single positive cells expressing genes indicated in green; magenta arrowhead: single positive cells expressing genes indicated in magenta. Magnified single confocal sections are shown for the dotted box area to the right of the image.

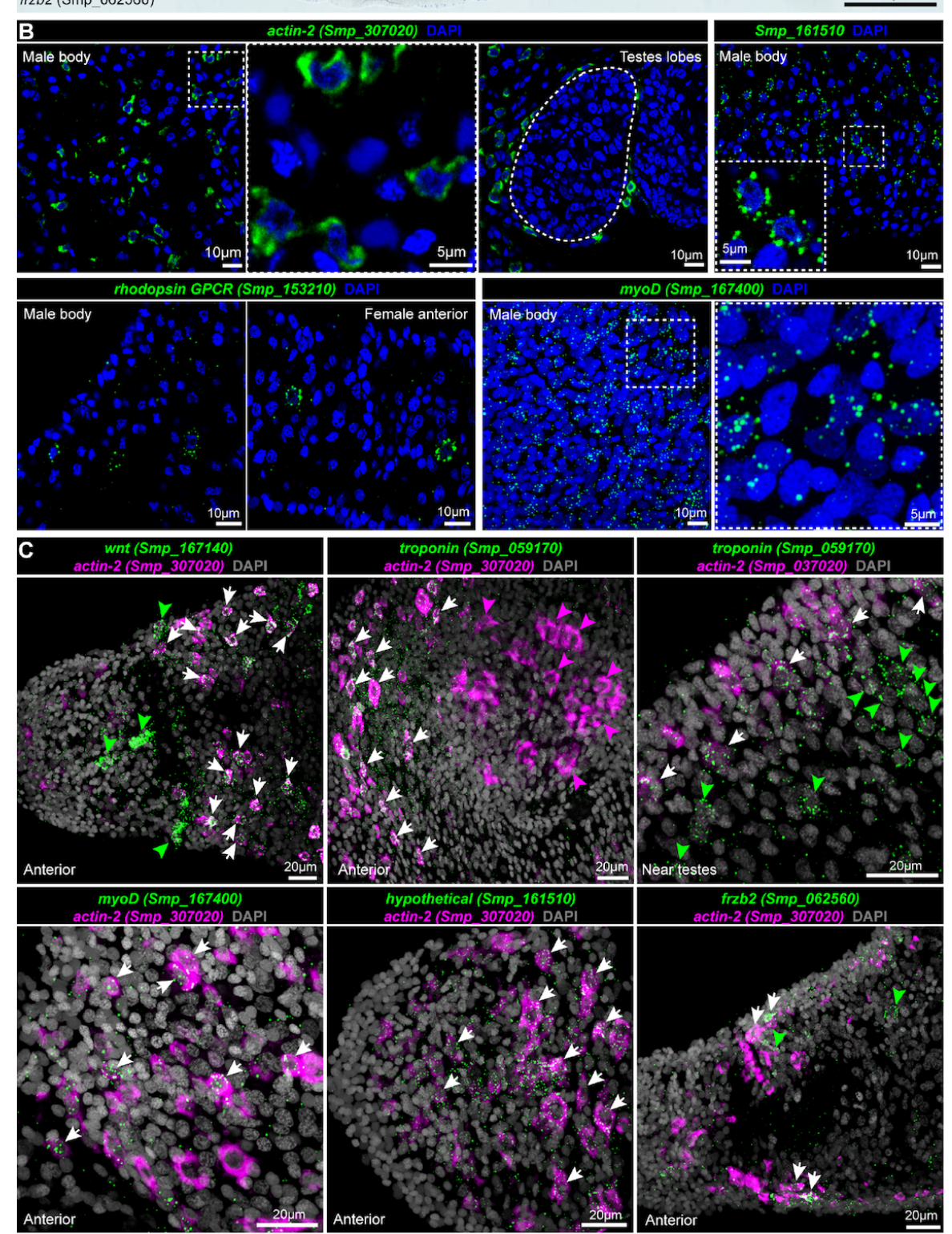


Supplementary Figure 3
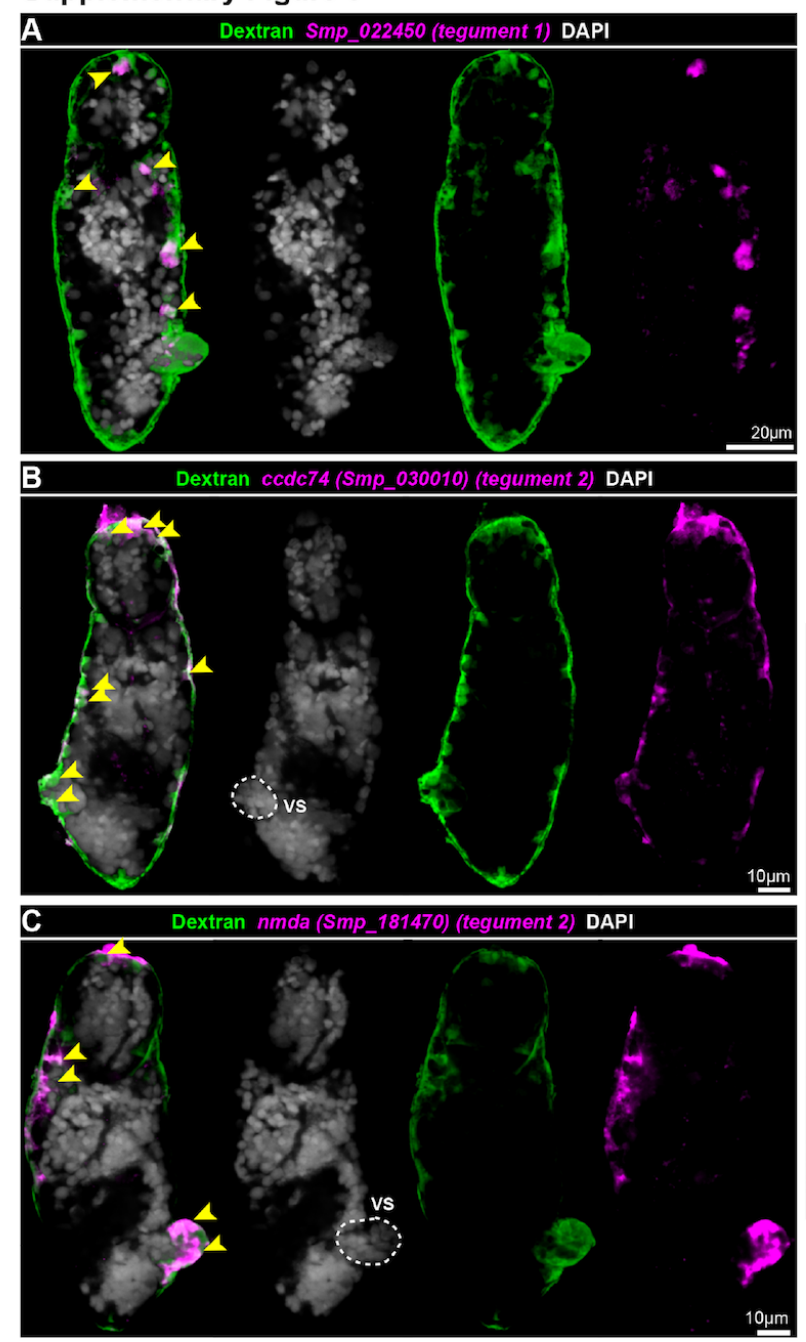

$F_{\text {st }}$

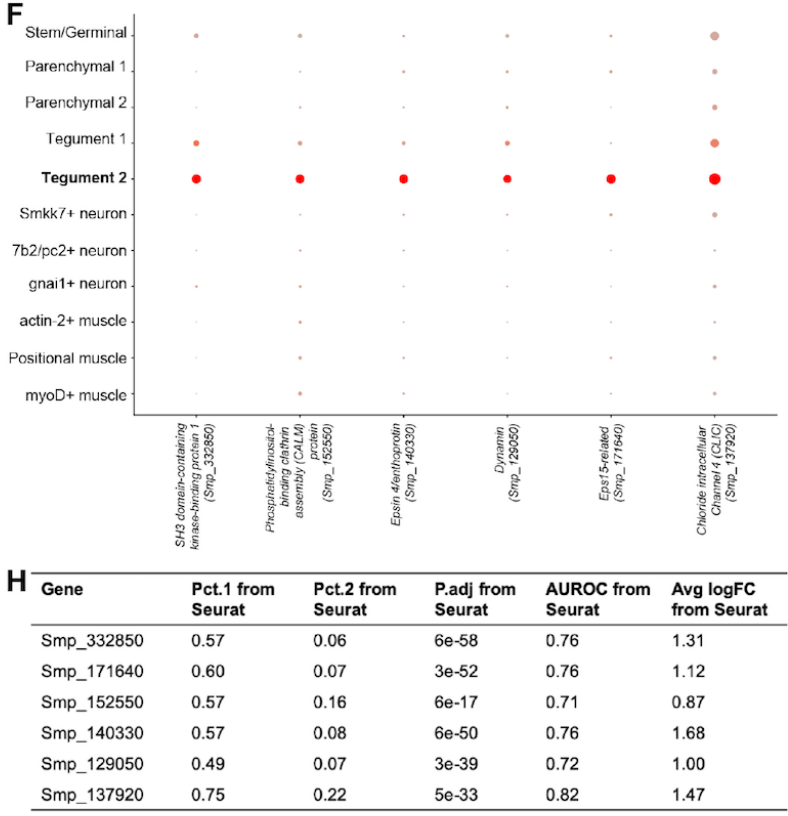

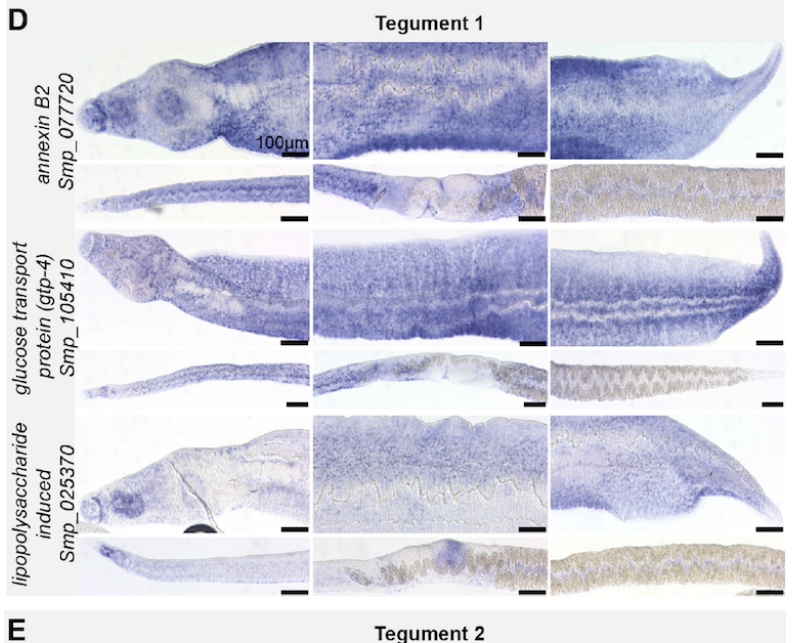
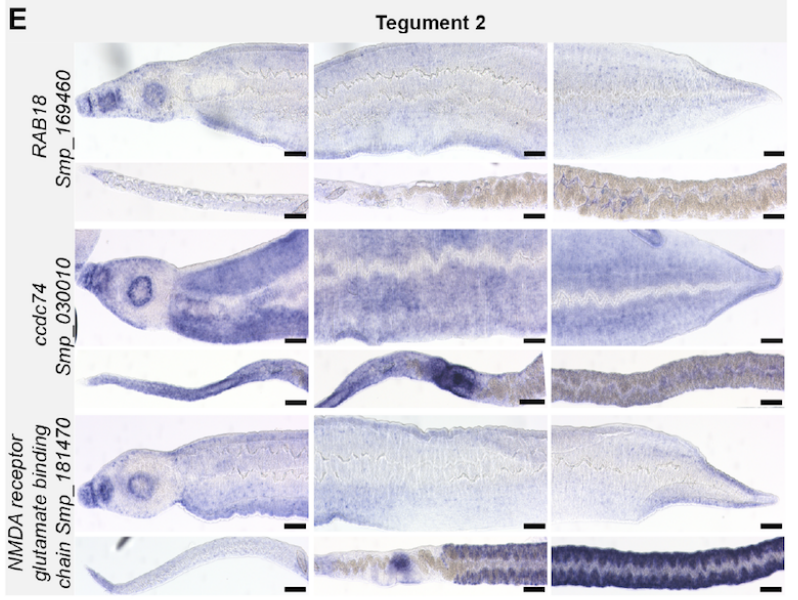

G

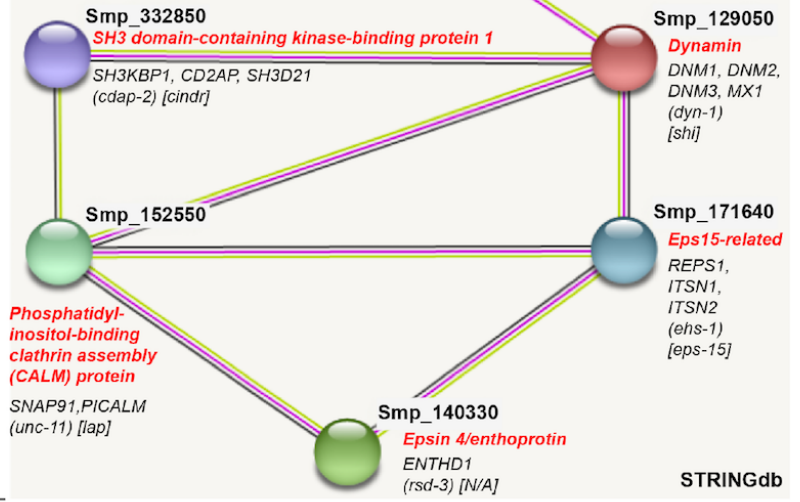

e- - from curated databases

Q- experimentallydetermined

$$
\begin{gathered}
\text { Others } \\
\text { Predicted Interactions } \\
\text { - gene neighborhood }
\end{gathered}
$$

Supplementary Figure 3. (A-C) Dextran labelling in schistosomula shows co-localisation with Tegument 1 and 2 markers. Yellow arrowheads indicate cells positive for both dextran and tegument marker. VS: ventral sucker. (D-E) WISH of Tegument 1 and Tegument 2 maker genes in adult parasites. Scale bar: $100 \mu \mathrm{m}$ (F) Expression profile of tegument 2 marker genes used for STRINGdb analysis. (G-H) Prediction of biological processes enriched in Tegument 2 relative to Tegument 1. We identified genes that are strong markers (with AUROC $\geq 0.7$ in Seurat) for Tegument 2 but not for Tegument 1 . The set of genes shown is the largest connected component, i.e. set of predicted interacting genes in the STRINGdb results. The interaction cluster included several genes related to clathrin-mediated (receptor-mediated) endocytosis. These included phosphatidylinositol-binding clathrin assembly protein (CALM), Eps15-related, and epsin-related genes. 
bioRxiv preprint doi: https://doi.org/10.1101/754713; this version posted September 11, 2019. The copyright holder for this preprint (which was not certified by peer review) is the author/funder, who has granted bioRxiv a license to display the preprint in perpetuity. It is made available under aCC-BY-NC-ND 4.0 International license.

\section{Supplementary Figure 4}
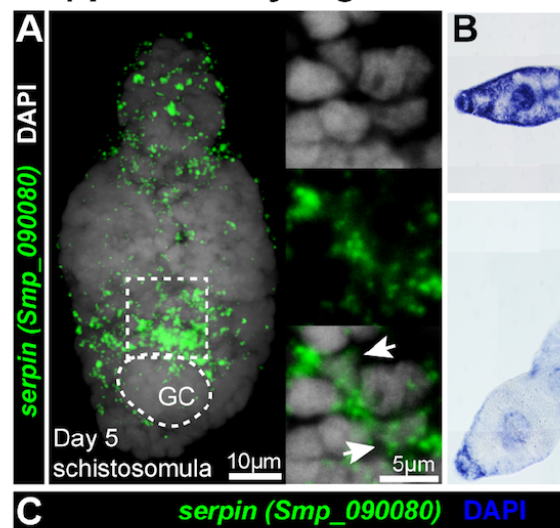

serpin (Smp_090080)

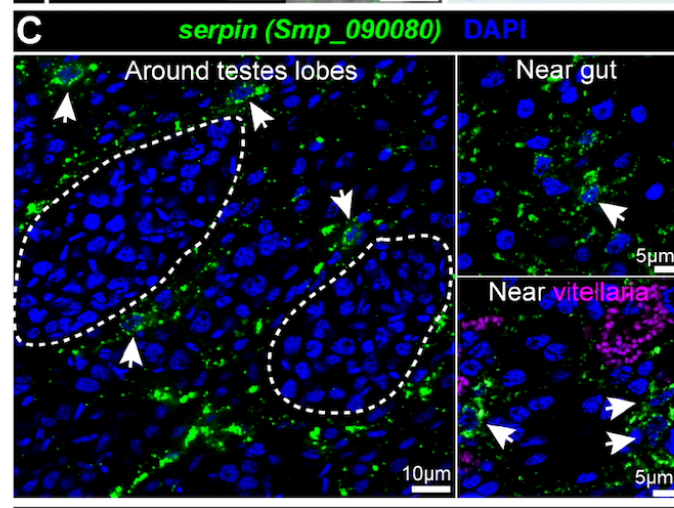

D $\quad$ LAP (Smp_030000)
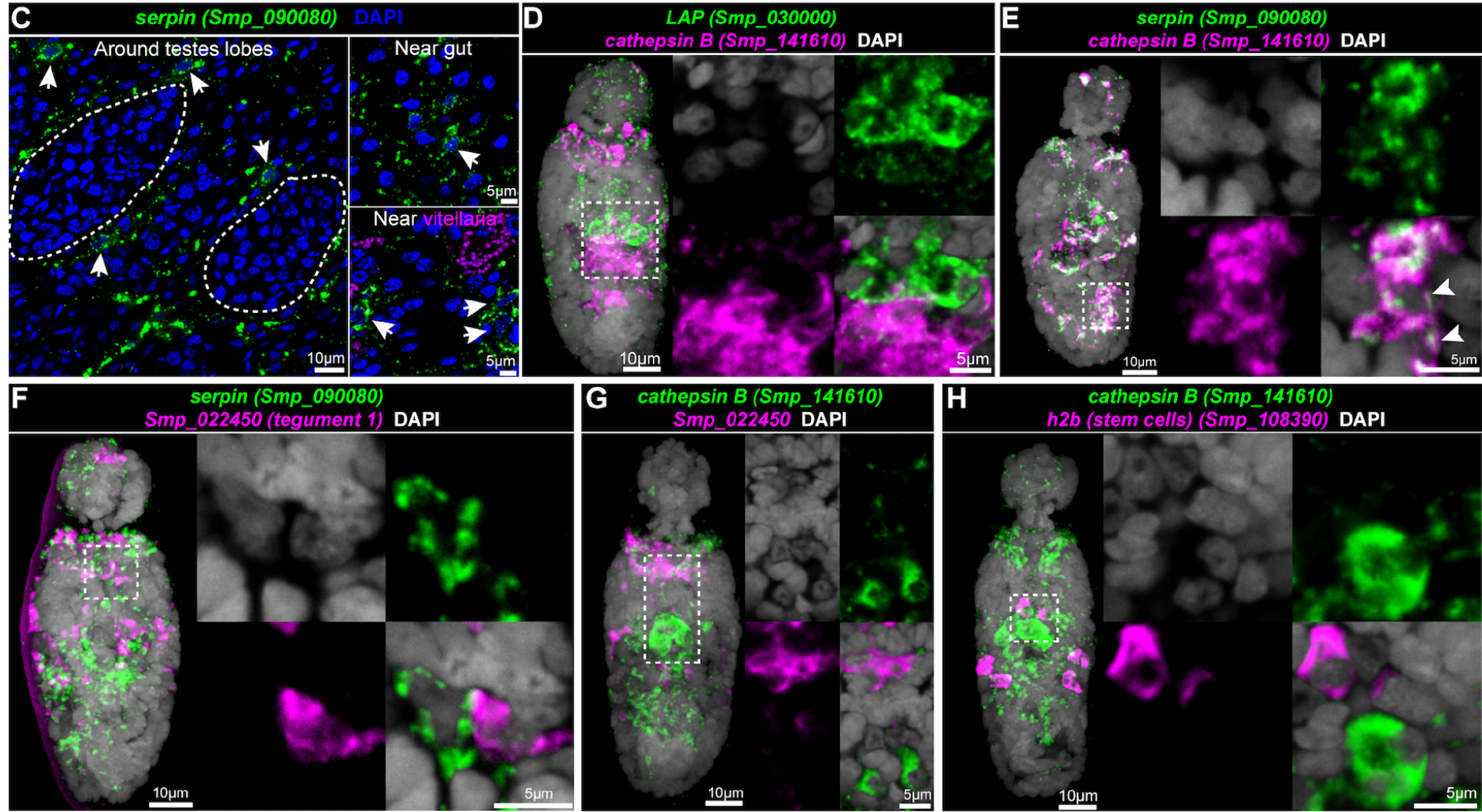

leucine aminopeptidase (LAP)(Smp_030000)
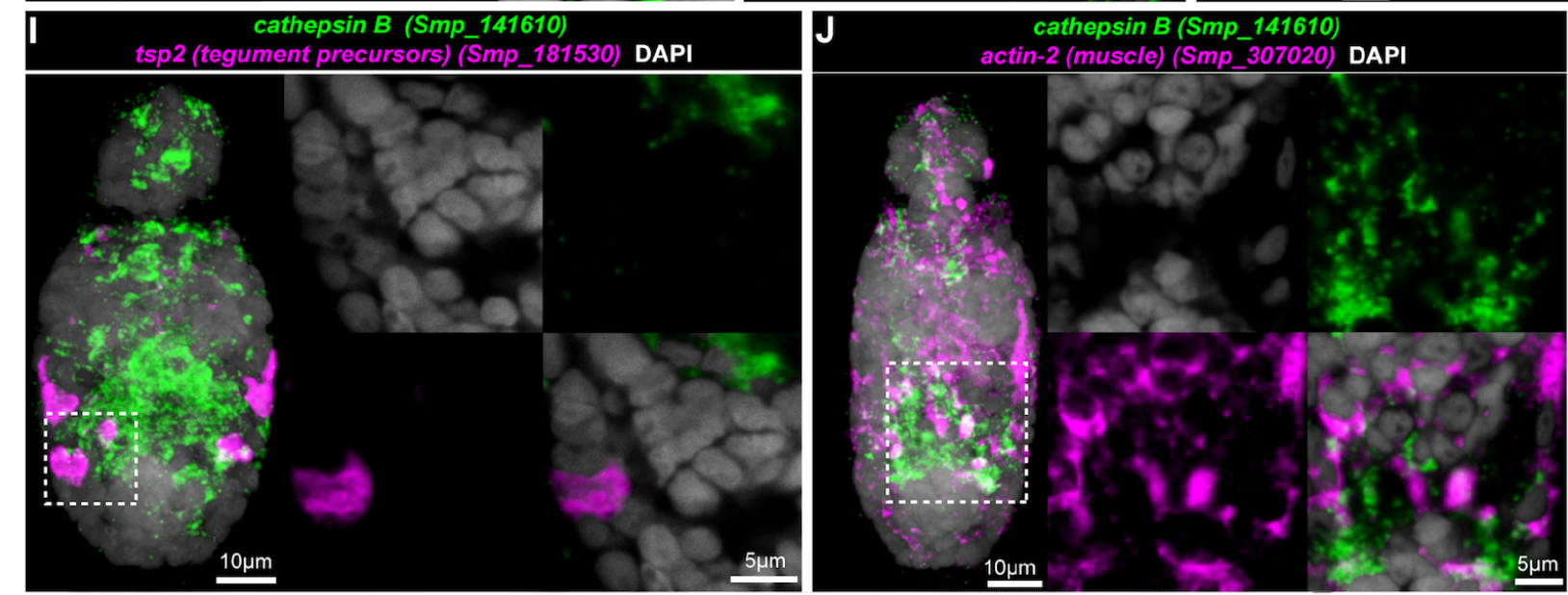

Supplementary Figure 4. (A) serpin FISH in five-day old schistosomula. MIP of whole worm is shown on the left, and single magnified confocal section from the dotted box is shown on the right. White arrows indicate a positive cell that has long cytoplasmic processes. (B) WISH of serpin and lap in adult parasites; lap is expressed in the worm parenchyma as well as in the gut. (C) Single confocal sections showing FISH of serpin in different regions of the worm. White arrows indicate single positive cells. (D-J) Double FISH of parenchymal cell markers and other indicated cell type markers in two-day old schistosomula. Parenchymal cell markers do not co-localise with the tegument cells (F-G), stem cells $(\mathrm{H})$, or tegument precursors (I) but show some co-localisation with muscle cells (J). MIP is shown for the whole worm on the left, and single confocal sections from the dotted box are shown on the right. 

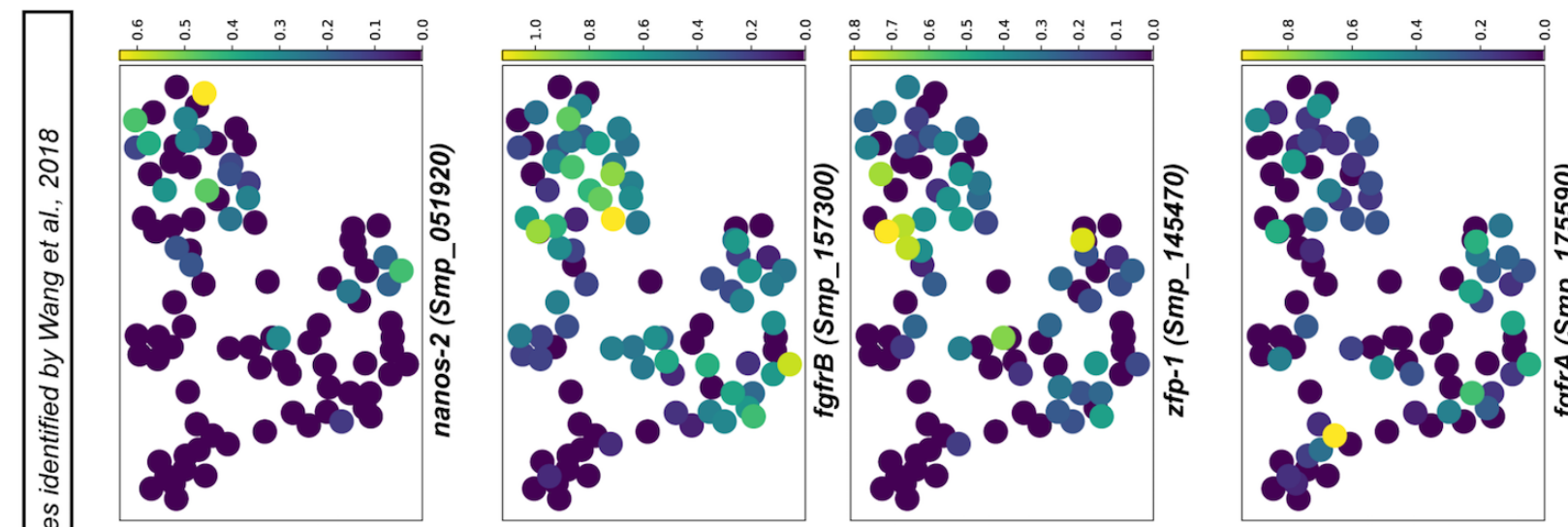

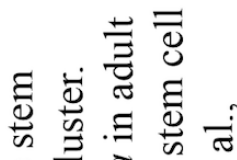

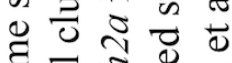

$\overline{0} \approx$ 政

营正苍

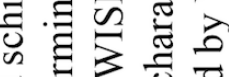

ত 苞导

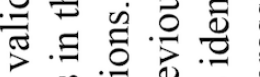

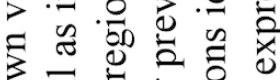
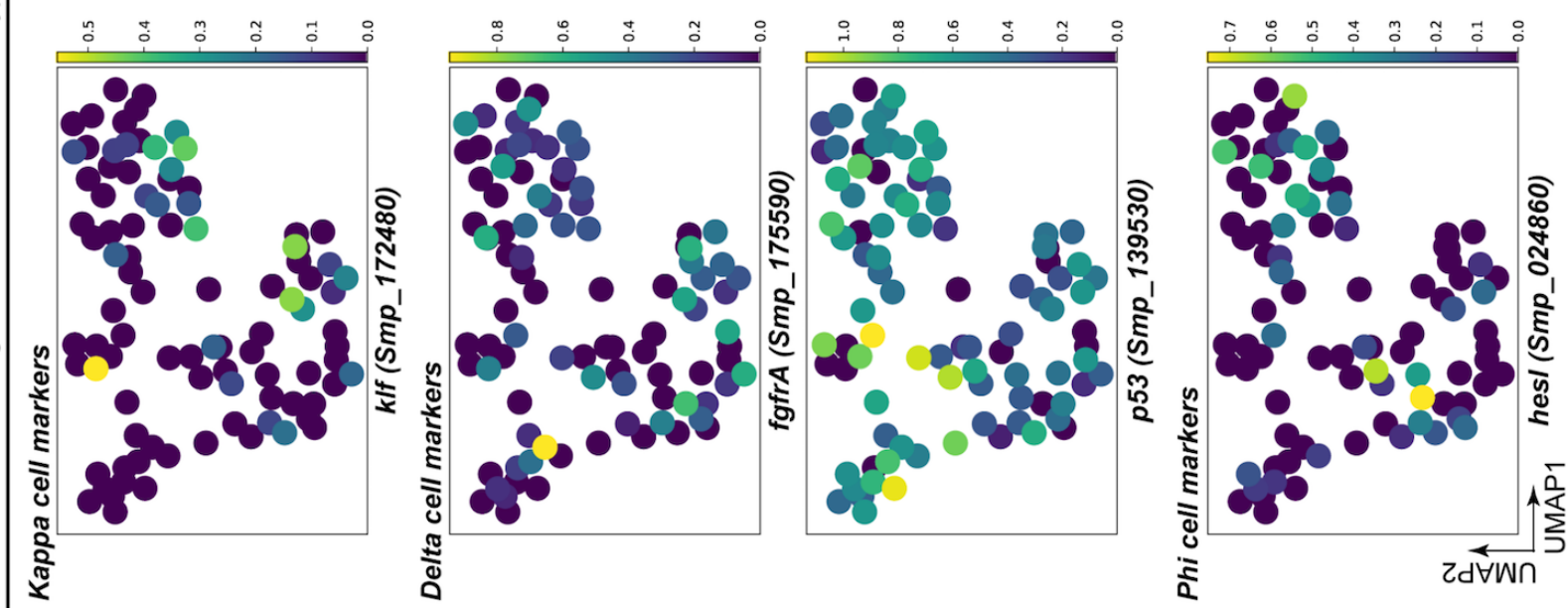

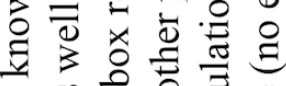

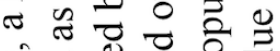

๙ิ

응 응

电式

I

施

䒕包

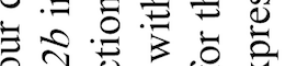

京.

ט.
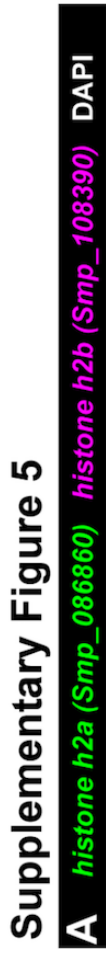
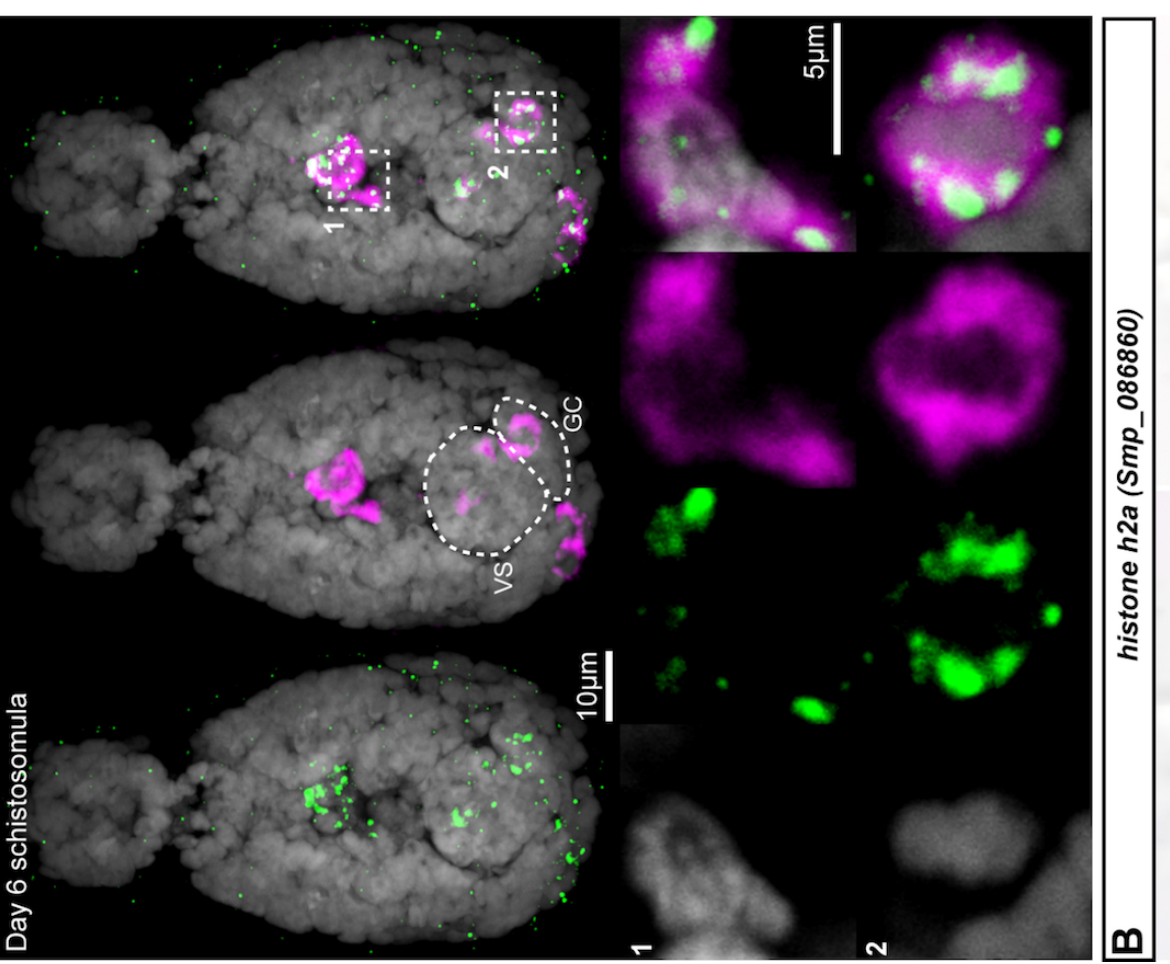

चु के च्ठ

这氜

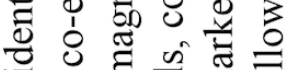

$\sigma \approx \approx \overline{8} \approx$

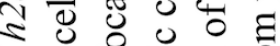

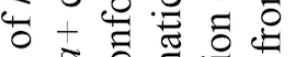

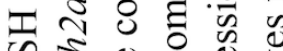

$1 \div \frac{0}{2}$ क

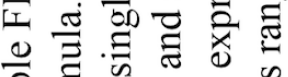

류

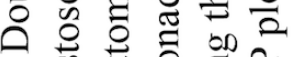

ช.

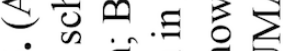

ம드 हี

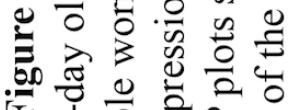

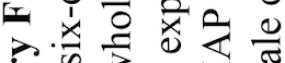

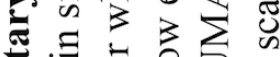

․ㅣㅇㅣ

㠃舫

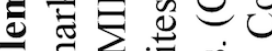

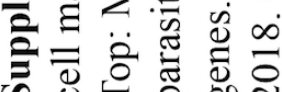


bioRxiv preprint doi: https://doi.org/10.1101/754713; this version posted September 11, 2019. The copyright holder for this preprint (which was not certified by peer review) is the author/funder, who has granted bioRxiv a license to display the preprint in perpetuity. It is made available under aCC-BY-NC-ND 4.0 International license.

\section{Supplementary Figure 6}

A

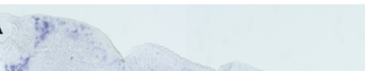

choline acetyltransferase (ChAT) (Smp_146910) Supplementary Figure 6.

(A) WISH of indicated neuronal markers in adult parasites. (B) FISH of Smp_203580 in adult male soma (mid-body) shows long cellular processes in each cell.

(C-D) Double FISH in adults reveals that (C) Smp_203580 does hypothetical (Smp_203580) not co-localise with pan-neuronal marker $7 b 2$, but (D) nearly all cells that express Smp 203580 co-express $\mathrm{Sm}-k \mathrm{k} 7$ (white signal).

Sm-kk7 (Smp_194830) (E-F) gnai is expressed throughout the body of the adult worm (E), but does not co-localise with Smp_203580 (F). Green and magenta arrowheads indicate single positive cells for respectively labelled genes.
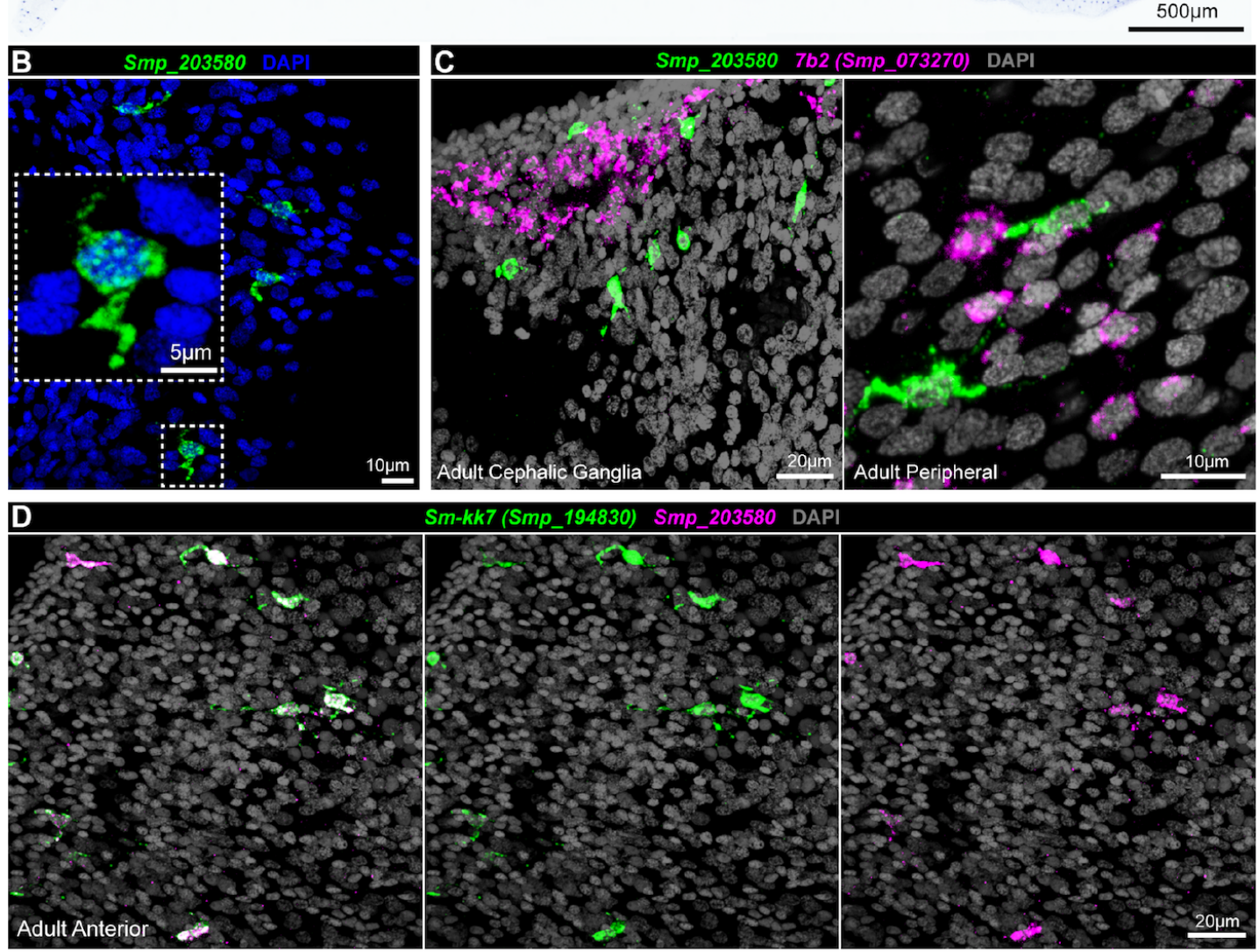

E

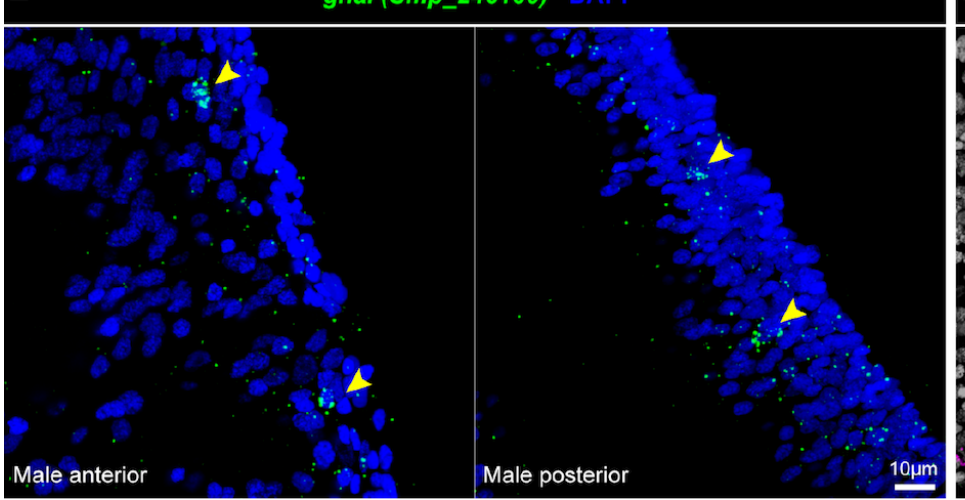

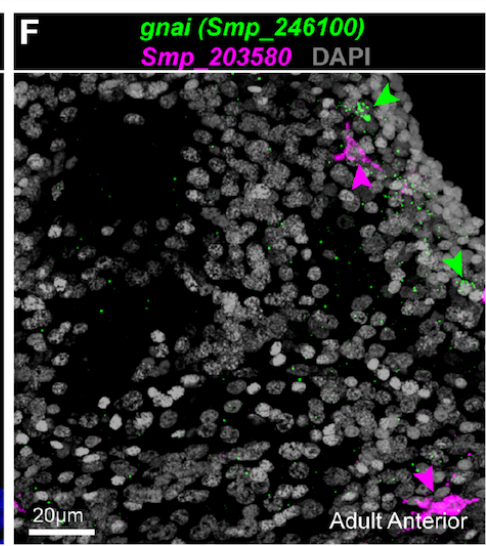

